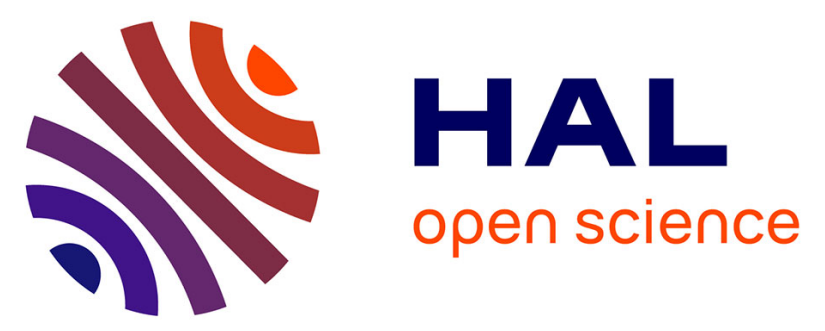

\title{
Reversal of Fe-Mg partitioning between garnet and staurolite in eclogite-facies metapelites from the Champtoceaux nappe (Brittany, France)
}

Michel Ballevre, J.L Pinardon, Jean Robert Kienast, Jean-Paul Vuichard

\section{- To cite this version:}

Michel Ballevre, J.L Pinardon, Jean Robert Kienast, Jean-Paul Vuichard. Reversal of Fe-Mg partitioning between garnet and staurolite in eclogite-facies metapelites from the Champtoceaux nappe (Brittany, France) . Journal of Petrology, 1989, 30 (6), pp.1321-1349. 10.1093/petrology/30.6.1321 . insu-01493270

\section{HAL Id: insu-01493270 \\ https://hal-insu.archives-ouvertes.fr/insu-01493270}

Submitted on 31 Mar 2017

HAL is a multi-disciplinary open access archive for the deposit and dissemination of scientific research documents, whether they are published or not. The documents may come from teaching and research institutions in France or abroad, or from public or private research centers.
L'archive ouverte pluridisciplinaire HAL, est destinée au dépôt et à la diffusion de documents scientifiques de niveau recherche, publiés ou non, émanant des établissements d'enseignement et de recherche français ou étrangers, des laboratoires publics ou privés. 


\title{
Reversal of Fe-Mg Partitioning Between Garnet and Staurolite in Eclogite-facies Metapelites from the Champtoceaux Nappe (Brittany, France)
}

\author{
by.MICHEL BALLEVRE ${ }^{1}$, JEAN-LUC PINARDON ${ }^{2}$, JEAN-ROBERT \\ KIENAST ${ }^{2}$ AND JEAN-PAUL VUICHARD ${ }^{1}$ \\ ${ }^{1}$ Laboratoire de tectonique, CAESS, Université de Rennes I, 35042 Rennes Cedex, France \\ ${ }^{2}$ Laboratoire de pétrologie métamorphique, U.A. C.N.R.S. 727, Université Paris VII, 75230 \\ Paris Cedex 05, France
}

(Received 29 June 1988; revised typescript accepted 24 January 1989)

\begin{abstract}
This paper concentrates on the petrology of eclogite-facies metapelites and, particularly, the significance of staurolite in these rocks.

A natural example of staurolite-bearing eclogitic micaschists from the Champtoceaux nappe (Brittany, France) is first described. The Champtoceaux metapelites present, in addition to quartz, phengite, and rutile, two successive parageneses: (1) chloritoid + staurolite + garnet cores, and (2) garnet rims + kyanite \pm chloritoid.

Detailed microprobe analyses show that garnet and chloritoid evolve towards more magnesian compositions and that staurolite is more Fe-rich than coexisting garnet. A comparison of the studied rocks with other known occurrences of eclogitic metapelites shows that whereas staurolite is always more Fe-rich than garnet in high-pressure eclogites, the reverse is true in low- to medium-pressure micaschists.

Phase relations between garnet, staurolite, chloritoid, biotite, and chlorite are analysed in the KFMASH system (with excess quartz, phengite, rutile, and $\mathrm{H}_{2} \mathrm{O}$ ). The topology of univariant reactions is depicted for a normal and a reverse $\mathrm{Fe}-\mathrm{Mg}$ partitioning between garnet and staurolite. Mineral compositional changes are also predicted for varying bulk-rock chemistries.

In the studied micaschists, the zonal arrangement of garnet inclusions and the progressive compositional changes of ferromagnesian phases record part of the prograde $\boldsymbol{P}-\boldsymbol{T}$ path, before the attainment of 'peak' metamorphic conditions (at about $650-700^{\circ} \mathrm{C}, 18-20 \mathrm{~kb}$ ). The retrograde path, which records the uplift of the Champtoceaux nappe, occurs under decreasing temperatures.
\end{abstract}

\section{INTRODUCTION}

Recent discoveries show that mineral assemblages in eclogitic metapelites are powerful tools for evaluating $P-T$ conditions (Chopin \& Schreyer, 1983; Koons \& Thompson, 1985; Goffé \& Chopin, 1986; Vuichard \& Ballèvre, 1988). Their use requires a theoretical knowledge of phase relations in the KFMASH multisystem and experimental data on the limiting KFASH or KMASH systems. In addition, careful examination of natural samples is necessary to depict actual phase relations.

Previous works (Harte \& Hudson, 1979; Koons \& Thompson, 1985; Vuichard \& Ballèvre, 1988 ) show that stable associations at relatively low temperatures (i.e., lower than about $600^{\circ} \mathrm{C}$ ) are garnet-chloritoid-chlorite and garnet-chloritoid-kyanite, and that garnetchloritoid-talc does not occur in pelitic rocks of 'normal' aluminous composition, i.e., intermediate to high $\mathrm{Fe} /(\mathrm{Fe}+\mathrm{Mg}$ ) ratio (Vuichard \& Ballèvre, 1988). At temperatures higher 
than about $600^{\circ} \mathrm{C}$, critical assemblages are garnet-chlorite-kyanite and garnet-talc-kyanite (Vuichard \& Ballèvre, 1988). Staurolite was not considered in detail in these previous studies.

The purpose of this paper is to discuss phase relations between garnet, chloritoid, staurolite, and kyanite in high-pressure metapelites. Staurolite-bearing eclogitic metapelites are known in Kazakhstan (Udovkina et al., 1980), the Dora-Maira nappe in the Western Alps (Chopin, 1985), the Fairbanks district of Alaska (Brown \& Forbes, 1986), the Hohe Tauern in the Eastern Alps (Spear \& Franz, 1986), the Najac klippe in the Massif Central, France (Delor et al., 1987), and the Champtoceaux nappe in Brittany, France (Ballèvre et al., 1987). Our discussion does not take into account the Hohe Tauern example because staurolite is stabilized there by high amounts of $\mathrm{ZnO}$ (Leupolt \& Franz, 1986; Spear \& Franz, 1986). In the five other occurrences, staurolite is a matrix phase in the Fairbanks and Najac micaschists and is observed only as inclusions within garnet in the Kazakhstan, DoraMaira and Champtoceaux micaschists.

In this paper, we will first describe the petrology of the Champtoceaux metapelites and compare them with other occurrences of eclogite-facies micaschists. We will show that a reversal of $\mathrm{Fe}-\mathrm{Mg}$ partitioning between garnet and staurolite occurs in high-pressure metapelites as previously suggested by Chopin (1985) and Vuichard \& Ballèvre (1988), and then explore the major consequences of this reversal for phase relations in the KFMASH multisystem.

\section{GEOLOGICAL SETTING}

The Champtoceaux nappe (Brittany, France) is located in the South Armorican domain between two major crustal faults: the Nort-sur-Erdre Fault to the north and the southern

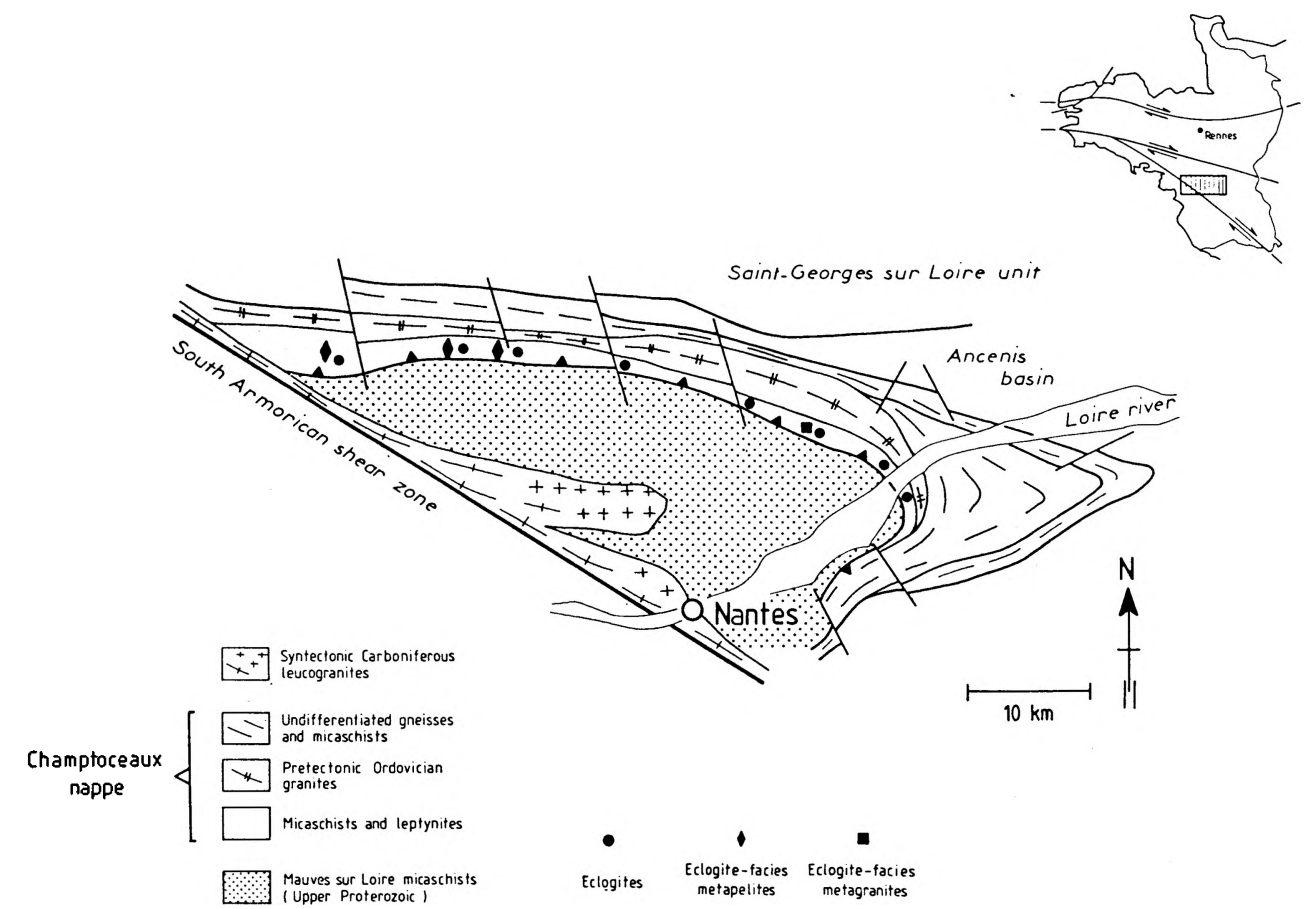

FIG. 1. Schematic structural map of the Champtoceaux nappe. Inset shows the location of the study area in Brittany (France). It should be noted that eclogite-facies metapelites are known only in the lower unit. 
branch of the South Armorican Shear Zone to the south (Fig. 1). The Champtoceaux nappe is thrust over low-grade metasediments of presumed Upper Proterozoic age. It consists of several superimposed units (Marchand, 1981), which underwent distinctive $P-T$ histories during the Variscan collisional event.

The lower unit of the Champtoceaux nappe consists essentially of leucocratic gneisses (i.e., leptynites) and micaschists which enclose numerous metabasic lenses. In general, the exact age of the protoliths is unknown, although some leptynites result from the intense deformation of granites (Lasnier et al., 1973; Lagarde, 1978) of probable Lower Paleozoic age (Vidal et al., 1980). The granites intruded sediments which locally preserve evidence of contact metamorphism (Marchand, 1983). The metabasites have basaltic whole-rock compositions and MORB-type REE patterns (Paquette, 1987). Relicts of primary igneous textures or minerals are lacking in the metabasites.

The lower unit of the Champtoceaux nappe suffered an eclogitic metamorphism at an early stage of the Variscan orogeny (Paquette et al., 1984, 1985; Paquette, 1987). Eclogitic parageneses are well known in the metabasic lenses (Lacroix, 1891; Brière, 1920; Velde, 1966, 1970; Godard et al., 1981; Paquette et al., 1985) but have been only recently dișcovered in the quartzites (Ballèvre et al., 1987). Garnet-kyanite assemblages in micaschists were first described by Lacroix (1891) but were attributed to the eclogitic episode much later (Ballevre et al., 1987). In addition, coronitic transformations in undeformed volumes of granitic rocks (Lasnier et al., 1973) could also be attributed to eclogite-facies rather than to granulite-facies metamorphism. Garnet-omphacite \pm kyanite associations from quartzites will be described fully elsewhere (Ballèvre and Kiénast, in preparation), and this paper concentrates on the petrology of the micaschists.

TABLE 1

List of mineral abbreviations used in text, figures, and tables

\begin{tabular}{ll}
\hline Abbreviations & Mineral names \\
\hline $\mathrm{Ab}$ & Albite \\
$\mathrm{Adr}$ & Andradite \\
$\mathrm{Alm}$ & Almandine \\
$\mathrm{An}$ & Anorthite \\
$\mathrm{And}$ & Andalusite \\
$\mathrm{Bt}$ & Biotite \\
$\mathrm{Chl}$ & Chlorite \\
$\mathrm{Cld}$ & Chloritoid \\
$\mathrm{Gr}$ & Graphite \\
$\mathrm{Gro}$ & Grossular \\
$\mathrm{Grt}$ & Garnet \\
$\mathrm{H}$ O & Vapour \\
$\mathrm{Ilm}$ & Ilmenite \\
$\mathrm{Jd}$ & Jadeite \\
$\mathrm{Ky}$ & Kyanite \\
$\mathrm{Phg}$ & Phengite \\
$\mathrm{Pg}$ & Paragonite \\
$\mathrm{Pyr}$ & Pyrope \\
$\mathrm{Qtz}$ & Quartz \\
$\mathrm{Rt}$ & Rutile \\
$\mathrm{Sil}$ & Sillimanite \\
$\mathrm{Spe}$ & Spessartine \\
$\mathrm{St}$ & Staurolite \\
$\mathrm{Tlc}$ & Talc \\
WM & White micas \\
\hline
\end{tabular}




\section{PETROGRAPHY}

Observed mineral parageneses in the Champtoceaux micaschists are listed in Table 2. All micaschists contain quartz, white mica, garnet, and rutile. In addition, kyanite is observed in some samples. Particular attention has been paid to garnet inclusions, notably chloritoid and staurolite, to define mineral assemblages during garnet growth (Table 2).

Most samples show evidence for ductile deformation during the growth of primary parageneses. In particular, garnet, kyanite, and sometimes white mica display numerous tiny inclusions of rutile \pm graphite, whose shape fabric defines an internal schistosity $S_{\mathrm{i}}$ (Spry, 1969). Staurolite and/or chloritoid inclusions within garnets are parallel to the $S_{i}$ (Figs. 2 and 3). The matrix foliation (i.e., external schistosity $S_{\mathrm{e}}$ ) is generally deformed by small-scale folds. Garnet and kyanite porphyroblasts have been submitted to rotations after their growth ceased. In consequence, $S_{\mathrm{i}}$ and $S_{\mathrm{e}}$ are no longer continuous. Kyanite and white mica also show evidence of intracrystalline deformation (undulose extinctions and kink-bands) (Lacroix, 1891).

A retrogressive overprint is generally present. In some samples, secondary phases grew in the absence of ductile deformation. For example, garnet is sometimess pseudomorphed by chlorite aggregates which preserve its shape and the alignment of rutile inclusions. Most samples suffered a ductile deformation during the retrograde history. In these cases, the overprint is generally more extensive. Shear bands nucleate on garnet or kyanite grains and a new foliation may develop; garnet, kyanite, and white mica act as porphyroclasts.

\section{Primary assemblages}

Primary metamorphic assemblages contain quartz, white mica, garnet, kyanite, and rutile. Microprobe analyses of white mica reveal that only phengite is present, except in samples CH13 and FAY26 where primary paragonite is also present. Talc was not observed. A detailed investigation of garnet-kyanite assemblages is presented in this study.

Garnet porphyroblasts commonly present inclusion-rich cores and inclusion-poor rims (Figs. 2 and 3). Garnet cores include quartz, phengite, rutile, and/or graphite. Garnet cores also include aluminous and ferromagnesian phases: chloritoid, staurolite or chloritoid + staurolite. Chloritoid shows a pale blue colour, a faint pleochroism, and sometimes polysynthetic twinning. Staurolite is colourless or pleochroic from pale yellow to yellow. Garnet rims contain kyanite, phengite, paragonite, and sometimes chloritoid, but never staurolite.

Kyanite porphyroblasts contain quartz, rutile \pm graphite, and white mica inclusions. Microprobe analyses reveal that most micas are phengites but paragonite was detected in sample CH13.

Chloritoid is observed in the matrix in samples FAY24, FAY26, and CH13 (Fig. 3). It is never in contact with quartz, garnet or kyanite and has irregular shapes. Matrix chloritoids have been partly 'dissolved' and are now observed as corroded relicts within white mica \pm chlorite aggregates.

To summarize, textural relations suggest the following successive parageneses (Figs. 2 and 3). During a first stage, garnet + chloritoid + staurolite assemblages crystallized. The second stage is related to the disappearance of chloritoid + staurolite and the growth of kyanite, leading to garnet-kyanite or garnet-chloritoid-kyanite assemblages. Garnetstaurolite-kyanite assemblages have never been observed. In garnet-chloritoid-kyanite assemblages, chloritoid also has been removed from the matrix assemblage, but is locally preserved within phengite \pm chlorite aggregates. 
TABLE 2

Observed primary parageneses in the Champtoceaux micaschists

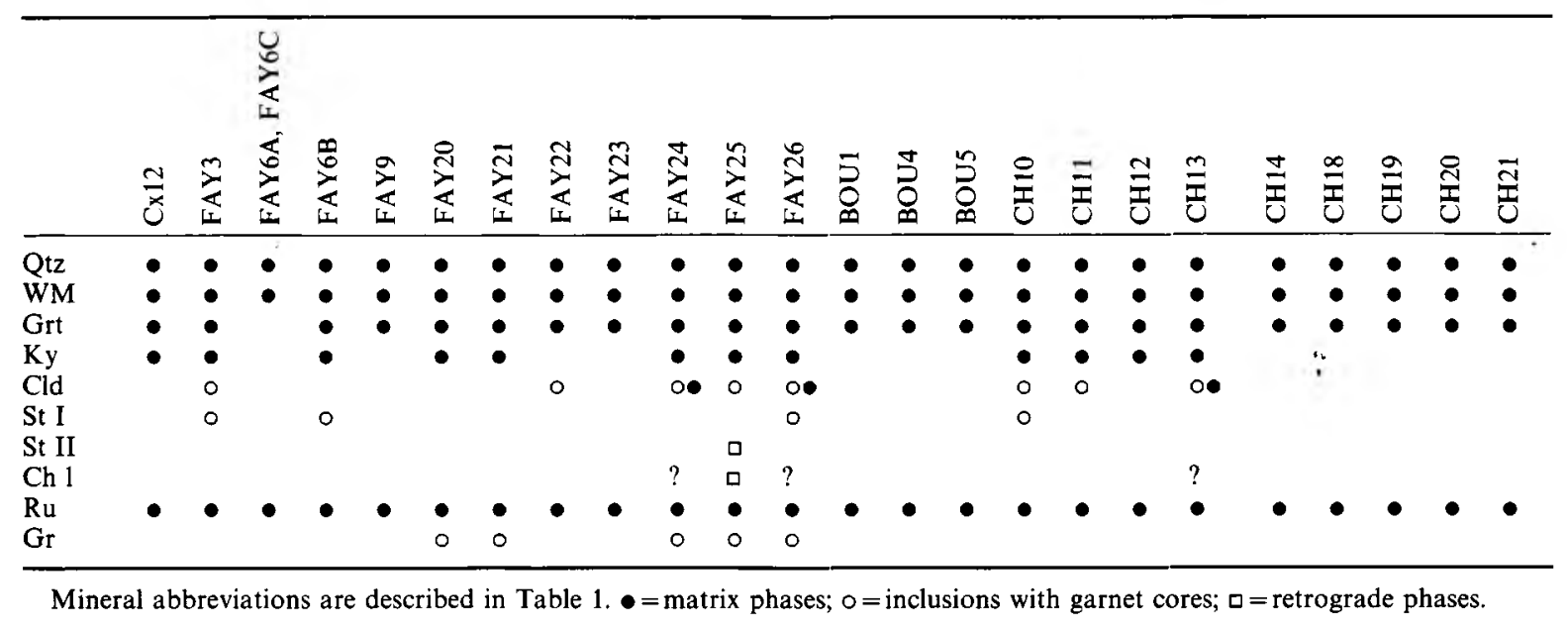




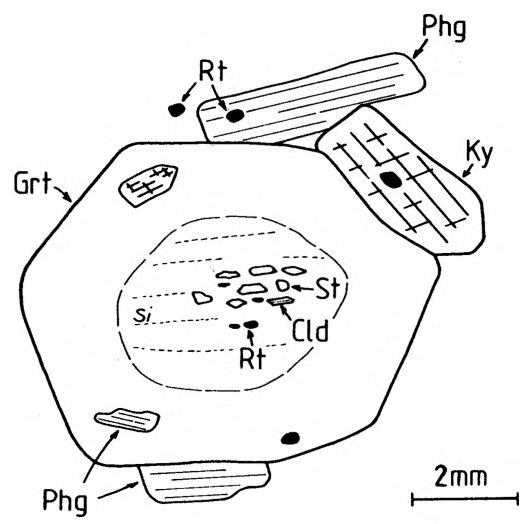

FIG. 2. Schematic textural relations between primary phases in the Champtoceaux micaschists (sample FAY3). Noteworthy features are the inclusion-rich core with internal schistosity $S_{\mathrm{i}}$ defined by minute graphite and rutile grains, and the progressive grain coarsening recorded by inclusions within garnet as compared with matrix grains. Mineral abbreviations are described in Table 1.

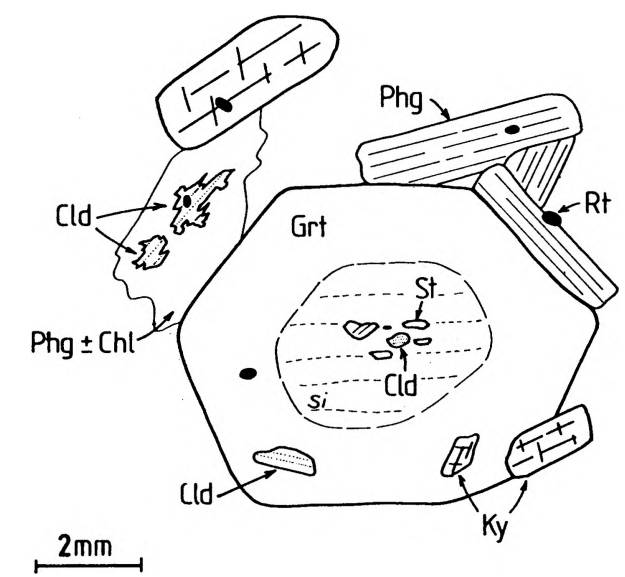

Fig. 3. Schematic textural relations between primary phases in the Champtoceaux micaschists (sample FAY26). Same remarks as for Fig. 2.

\section{Secondary assemblages}

Secondary phases are only locally developed and have the following features:

(1) Staurolite and chloritoid inclusions are frequently replaced by fine-grained aggregates containing chlorite, paragonite, margarite, kyanite, and ilmenite. Chlorite, paragonite, and margarite are intergrown.

(2) Garnet is sometimes pseudomorphed by chlorite aggregates containing zircon grains with pleochroic aureoles (Lacroix, 1891).

(3) Kyanite inclusions and porphyroblasts are rimmed by fine-grained micaceous aggregates.

(4) Large primary phengites are locally destabilized into finer-grained secondary phengites.

(5) In samples FAY26 and FAY24, rare plagioclase grains have grown in the matrix or in garnet cracks. 
(6) In rare instances (sample FAY25), staurolite II + chlorite has grown at the expense of garnet + kyanite. Staurolite II grains are euhedral or subhedral, whereas staurolite grains included in garnets are anhedral.

\section{MINER ALOGY}

Garnet- and kyanite-bearing micaschists were selected with no or little retrograde overprint. Mineral analyses were performed on five samples (FAY3, FAY24, FAY25, FAY26, and CH10) with a Camebax microprobe analyser (Paris VI University). Operating conditions were: $15 \mathrm{kV}$ accelerating voltage, $10 \mathrm{nA}$ probe current and $10 \mathrm{~s}$ counting-time. Standards were: albite $(\mathrm{Na})$, orthoclase $(\mathrm{K}, \mathrm{Al})$, diopside $(\mathrm{Ca}, \mathrm{Mg}, \mathrm{Si}), \mathrm{MnTiO}_{3}(\mathrm{Mn}, \mathrm{Ti})$, $\mathrm{Fe}_{2} \mathrm{O}_{3}(\mathrm{Fe})$ and $\mathrm{ZnS}(\mathrm{Zn})$. $\mathrm{Zn}$ was detected with the $\mathrm{L}_{\alpha}$ ray, and all others using the $\mathrm{K}_{\alpha}$ ray. Micas and plagioclases were analysed with an enlarged beam to avoid alkali loss. Additional information on mineral compositions has been obtained with the 'Microsonde Ouest' (Brest) on samples $\mathrm{Cx} 12$ and $\mathrm{CH} 13$.

\section{Garnet (Table 3)}

In all the analysed micaschists, garnets have low $\mathrm{CaO}$ and $\mathrm{MnO}$ contents and are zoned. From core to rim, they display a decrease in $\mathrm{MnO}$ and $\mathrm{CaO}$ content, an increasing $\mathrm{MgO}$ content and the $\mathrm{FeO}$ content remains approximately constant. In consequence, the $\mathrm{Mg} / \mathrm{Fe}$ $+\mathrm{Mg}$ ) ratio $\left(X_{\mathrm{Mg}}\right)$ increases from 0.10 to 0.25 (Fig. 4). Small chemical differences between garnet rims from different samples are observed.

(1) The grossular content of garnet rims is negligible in sample FAY26 ( $2 \mathrm{~mol} . \%)$ but attains up to $9 \mathrm{~mol} . \%$ in sample FAY3 (Table 2).

(2) The $X_{\mathrm{Mg}}$ ratio is higher in samples FAY24 and FAY26 (0.25-0.27) than in samples FAY3 and $\mathrm{CH} 10(0 \cdot 22-0 \cdot 25)$.

Chemical gradients within garnet grains are smoother in staurolite + chloritoid-bearing garnet cores than in staurolite-free rims. Moreover, in samples FAY26 and CH13, where chloritoid occurs in garnet rims and in the matrix, the magnesium enrichment in garnet is stronger than in samples FAY3 and $\mathrm{CH} 10$, where only kyanite is observed in garnet rims (Fig. 4).

\section{Chloritoid (Table 4)}

Chloritoid grains show trace amounts of $\mathrm{TiO}_{2}$ and $\mathrm{ZnO}$, very low $\mathrm{MnO}$ content, and no $\mathrm{Al}_{2} \mathrm{O}_{3}$ variation. Calculated $\mathrm{Fe}_{2} \mathrm{O}_{3}$ content is generally very low ( $1 \mathrm{wt} . \%$ or less). The main chemical variation is due to the $\mathrm{FeMg}_{-1}$ substitution.

In staurolite-chloritoid garnet cores, chloritoid grains are less magnesian in the inner zone $\left(X_{\mathrm{Mg}}=0 \cdot 20\right)$ than in the outer zone $\left(X_{\mathrm{Mg}}=0.25\right)$.

In samples FAY24, FAY26, and CH13, chloritoid inclusions in the garnet rim and matrix chloritoids have similar $X_{\mathrm{Mg}}$ (near 0.3). As for garnet, the magnesium enrichment is stronger in samples FAY26 and $\mathrm{CH} 13$ than in samples FAY3 and $\mathrm{CH} 10$. These observations show that the disappearance of the staurolite + chloritoid assemblage is accompanied by a strong $X_{\mathrm{Mg}}$ increase in the remaining ferromagnesian phases, i.e., garnet and chloritoid.

\section{Staurolite (Table 5)}

Following Pigage \& Greenwood (1982), we adopted the formula $\mathrm{Fe}_{2} \mathrm{Al}_{9} \mathrm{Si}_{3 \cdot 75} \mathrm{O}_{22}(\mathrm{OH})_{2}$ for staurolite and calculated the structural formulae on a basis of 23 oxygens. 
TABLE 3

Representative garnet analyses and structural formulae on a basis of 12 oxygens

\begin{tabular}{|c|c|c|c|c|c|c|c|}
\hline & $l$ & 2 & 3 & 4 & 5 & 6 & 7 \\
\hline \multicolumn{8}{|c|}{ Sample FAY3 } \\
\hline $\mathrm{SiO}_{2}$ & $37 \cdot 36(0 \cdot 37)$ & $37 \cdot 36(0 \cdot 26)$ & $36.75(0.04)$ & $37.48(0.08)$ & $37.61(0.04)$ & $37.73(0.03)$ & $37.77(0.04)$ \\
\hline $\mathrm{TiO}_{2}$ & $0.07(0.01)$ & $0.05(0.02)$ & $0.05(0.04)$ & $0.10(0.01)$ & $0.05(0.02)$ & $0.05(0.04)$ & $0.05(0.02)$ \\
\hline $\mathrm{Al}_{2} \mathrm{O}_{3}$ & $20.93(0.06)$ & $20 \cdot 39(0 \cdot 22)$ & $20 \cdot 48(0.40)$ & $21 \cdot 10(0 \cdot 04)$ & $21.22(0.04)$ & $21.34(0.07)$ & $21.08(0.04)$ \\
\hline $\mathrm{Cr}_{2} \mathrm{O}_{3}$ & $0.00(0-00)$ & $0.00(0.00)$ & $0.00(0-00)$ & $0.00(0.00)$ & $0.00(0.00)$ & $0.00(0-00)$ & $0.00(0.00)$ \\
\hline $\mathrm{Fe}_{2} \mathrm{O}_{3}$ & $0 \cdot 15(0 \cdot 22)$ & $0.61(0.28)$ & $0.45(0.12)$ & $0.00(0-00)$ & $0.00(0.00)$ & $0.00(0-00)$ & $0.00(0.00)$ \\
\hline $\mathrm{FeO}$ & $30 \cdot 64(0 \cdot 34)$ & $30 \cdot 27(0 \cdot 39)$ & $30.34(0.34)$ & $30.56(0.03)$ & $30 \cdot 42(0 \cdot 18)$ & $30 \cdot 17(0 \cdot 17)$ & $30-41(0-11)$ \\
\hline $\mathrm{MnO}$ & $3.08(0 \cdot 60)$ & $2 \cdot 91(0-34)$ & $2 \cdot 02(0 \cdot 14)$ & $1.65(0.23)$ & $1 \cdot 21(0 \cdot 04)$ & $1.22(0.06)$ & $1 \cdot 13(0.02)$ \\
\hline $\mathrm{MgO}$ & $2 \cdot 36(0 \cdot 17)$ & $2 \cdot 49(0 \cdot 15)$ & $2 \cdot 64(0 \cdot 15)$ & $2.72(0.05)$ & $3 \cdot 96(0 \cdot 11)$ & $4 \cdot 74(0 \cdot 15)$ & $5.07(0.08)$ \\
\hline $\mathrm{CaO}$ & $5.00(0.28)$ & $5.03(0.32)$ & $5.29(0.27)$ & $5.44(0.04)$ & $4.57(0.11)$ & $3 \cdot 76(0 \cdot 16)$ & $3 \cdot 37(0 \cdot 11)$ \\
\hline$\Sigma$ oxides & $99.59(0.29)$ & $99 \cdot 07(0.04)$ & $97.96(0.57)$ & $99.03(0.15)$ & $99 \cdot 04(0.12)$ & $99.00(0.12)$ & $98 \cdot 85(0 \cdot 21)$ \\
\hline Si & 3.006 & 3.020 & 3.001 & $3 \cdot 014$ & 3.007 & 3.006 & $3 \cdot 013$ \\
\hline $\mathrm{Al}^{\mathrm{IV}}$ & 0.000 & 0.000 & 0.000 & 0.000 & 0.000 & 0.000 & 0.000 \\
\hline$\Sigma^{\mathbf{l V}}$ & 3.006 & $3 \cdot 020$ & 3.001 & $3 \cdot 014$ & 3.007 & $3 \cdot 006$ & 3.013 \\
\hline $\mathrm{Al}^{\mathrm{VI}}$ & 1.986 & 1.944 & 1.971 & $2 \cdot 000$ & 2.000 & 2.005 & 1.982 \\
\hline $\mathrm{Ti}$ & 0.004 & 0.003 & 0.003 & $0 \cdot 006$ & 0.003 & $0 \cdot 003$ & $0 \cdot 003$ \\
\hline $\mathrm{Cr}$ & 0.000 & $0 \cdot 000$ & 0.000 & 0.000 & 0.000 & 0.000 & 0.000 \\
\hline $\mathrm{Fe}^{3+}$ & 0.009 & 0.037 & 0.027 & 0.000 & 0.000 & 0.000 & 0.000 \\
\hline$\Sigma^{\mathrm{VI}}$ & 2.000 & 1.984 & 2.002 & $2 \cdot 006$ & $2 \cdot 003$ & 2.007 & 1.985 \\
\hline $\mathrm{Fe}^{2+}$ & $2 \cdot 062$ & $2 \cdot 047$ & $2 \cdot 072$ & $2 \cdot 055$ & $2 \cdot 034$ & $2 \cdot 011$ & $2 \cdot 029$ \\
\hline $\mathrm{Mn}$ & 0.210 & $0 \cdot 200$ & 0.140 & $0 \cdot 112$ & 0.082 & $0 \cdot 082$ & $0 \cdot 076$ \\
\hline $\mathrm{Mg}$ & 0.283 & $0 \cdot 300$ & 0.321 & 0.325 & 0.472 & 0.562 & 0.602 \\
\hline $\mathrm{Ca}$ & 0.431 & 0.436 & 0.463 & 0.468 & $0 \cdot 392$ & 0.321 & 0.288 \\
\hline$\Sigma^{2+}$ & $2 \cdot 986$ & $2-982$ & 2.995 & $2 \cdot 961$ & $2 \cdot 980$ & 2.976 & 2.995 \\
\hline $\mathrm{Mg} /(\mathrm{Mg}+\mathrm{Fe})$ & $0 \cdot 12$ & $0 \cdot 13$ & 0.13 & $0 \cdot 14$ & $0 \cdot 19$ & $0 \cdot 22$ & $0-23$ \\
\hline Alm & $69 \cdot 07$ & $68 \cdot 64$ & $69 \cdot 17$ & $69 \cdot 41$ & 68.27 & 67.56 & $67 \cdot 74$ \\
\hline Pyr & $9 \cdot 48$ & $10 \cdot 06$ & $10 \cdot 71$ & $10 \cdot 99$ & $15 \cdot 84$ & 18.90 & $20 \cdot 11$ \\
\hline Spe & 7.02 & $6 \cdot 69$ & 4.68 & $3 \cdot 78$ & $2 \cdot 75$ & $2 \cdot 77$ & $2 \cdot 54$ \\
\hline Gro & $13 \cdot 74$ & $12 \cdot 38$ & 13.93 & $15 \cdot 52$ & $13 \cdot 00$ & $10 \cdot 64$ & 8.89 \\
\hline Adr & 0.46 & $1 \cdot 34$ & 1.01 & $0 \cdot 19$ & 0.09 & 0.09 & 0.09 \\
\hline
\end{tabular}




\begin{tabular}{|c|c|c|c|c|c|c|c|}
\hline \multicolumn{8}{|c|}{ Sample $\mathrm{CH} 10$} \\
\hline $\mathrm{SiO}_{2}$ & $35 \cdot 77(0 \cdot 46)$ & $36 \cdot 30(0 \cdot 25)$ & $36.67(0.06)$ & $37 \cdot 26(0 \cdot 27)$ & $37.56(0.03)$ & $37 \cdot 15(0 \cdot 27)$ & $36.94(0 \cdot 17)$ \\
\hline $\mathrm{TiO}_{2}$ & $0.06(0.04)$ & $0.05(0.02)$ & $0 \cdot 15(0 \cdot 16)$ & $0.07(0.07)$ & $0 \cdot 12(0 \cdot 01)$ & $0.03(0.05)$ & $0.07(0.01)$ \\
\hline $\mathrm{Al}_{2} \mathrm{O}_{3}$ & $21 \cdot 25(0 \cdot 17)$ & $21 \cdot 34(0.05)$ & $21.62(0.05)$ & $21 \cdot 60(0 \cdot 18)$ & $21.85(0.06)$ & $21 \cdot 71(0 \cdot 26)$ & $21 \cdot 76(0 \cdot 21)$ \\
\hline $\mathrm{Cr}_{2} \mathrm{O}_{3}$ & $0.00(0.00)$ & $0.00(0.00)$ & $0.00(0.00)$ & $0.00(0.00)$ & $0.00(0.00)$ & $0.00(0.00)$ & $0.00(0.00)$ \\
\hline $\mathrm{Fe}_{2} \mathrm{O}_{3}$ & $1 \cdot 34(0 \cdot 18)$ & $0.65(0.07)$ & $1.01(0.40)$ & $0 \cdot 40(0 \cdot 19)$ & $0 \cdot 40(0 \cdot 56)$ & $0.81(0.56)$ & $0 \cdot 86(0 \cdot 57)$ \\
\hline $\mathrm{FeO}$ & $30.90(0 \cdot 39)$ & $32 \cdot 28(0 \cdot 70)$ & $32 \cdot 15(0 \cdot 28)$ & $31.99(0.26)$ & $30.93(0.41)$ & $30.90(1.00)$ & $30.65(0.22)$ \\
\hline $\mathrm{MnO}$ & $4 \cdot 16(0 \cdot 48)$ & $2 \cdot 03(0 \cdot 13)$ & $1.01(0.05)$ & $0.89(0.08)$ & $0.99(0.06)$ & $1 \cdot 13(0.09)$ & $0.97(0.07)$ \\
\hline $\mathrm{MgO}$ & $2 \cdot 30(0 \cdot 15)$ & $2 \cdot 75(0.08)$ & $3.53(0.57)$ & $3.69(0 \cdot 31)$ & $4 \cdot 98(0 \cdot 36)$ & $5 \cdot 01(0 \cdot 18)$ & $5 \cdot 83(0 \cdot 48)$ \\
\hline $\mathrm{CaO}$ & $4 \cdot 19(0 \cdot 88)$ & $4 \cdot 17(0 \cdot 68)$ & $4 \cdot 61(0 \cdot 46)$ & $4 \cdot 57(0 \cdot 27)$ & $3.95(0.01)$ & $3.60(0 \cdot 83)$ & $2.75(0.08)$ \\
\hline$\Sigma$ oxides & $99.96(0.79)$ & $99 \cdot 55(0 \cdot 21)$ & $100 \cdot 72(0 \cdot 10)$ & $100 \cdot 45(0 \cdot 46)$ & $100 \cdot 76(0 \cdot 58)$ & $100 \cdot 34(0 \cdot 70)$ & $99 \cdot 83(1 \cdot 23)$ \\
\hline $\mathrm{Si}$ & $2 \cdot 900$ & 2.935 & $2 \cdot 917$ & $2 \cdot 958$ & $2 \cdot 951$ & $2 \cdot 937$ & $3 \cdot 013$ \\
\hline $\mathrm{Al}^{\mathrm{IV}}$ & $0 \cdot 100$ & 0.065 & 0.083 & 0.042 & 0.049 & 0.063 & 0.000 \\
\hline$\Sigma^{\text {IV }}$ & 3.000 & 3.000 & 3.000 & 3.000 & 3.000 & 3.000 & $3 \cdot 013$ \\
\hline $\mathrm{Al}^{\mathrm{VI}}$ & 1.931 & 1.969 & 1.944 & 1.979 & 1.975 & 1.961 & 1.982 . \\
\hline $\mathrm{Ti}$ & 0.003 & 0.003 & 0.009 & 0.004 & 0.007 & 0.002 & 0.003 \\
\hline $\mathrm{Cr}$ & 0.000 & 0.000 & 0.000 & 0.000 & 0.000 & 0.000 & 0.000 \\
\hline $\mathrm{Fe}^{3+}$ & 0.082 & $0 \cdot 040$ & 0.060 & 0.024 & 0.023 & $0 \cdot 048$ & 0.000 \\
\hline$\Sigma^{\mathrm{VI}}$ & $2 \cdot 017$ & $2 \cdot 011$ & $2 \cdot 013$ & 2.007 & 2.005 & $2 \cdot 011$ & 1.985 \\
\hline $\mathrm{Fe}^{2+}$ & 2.096 & $2 \cdot 183$ & $2 \cdot 139$ & $2 \cdot 124$ & $2 \cdot 033$ & 2.043 & 2.029 \\
\hline $\mathrm{Mn}$ & $0 \cdot 286$ & $0 \cdot 139$ & 0.068 & 0.060 & 0.066 & 0.076 & 0.076 \\
\hline $\mathrm{Mg}$ & $0 \cdot 278$ & 0.331 & 0.419 & 0.436 & 0.583 & 0.590 & 0.602 \\
\hline $\mathrm{Ca}$ & 0.364 & $0 \cdot 361$ & 0.393 & 0.389 & 0.333 & 0.305 & 0.288 \\
\hline$\Sigma^{2+}$ & 3.023 & $3 \cdot 014$ & $3 \cdot 018$ & 3.009 & 3.014 & 3.015 & $2 \cdot 995$ \\
\hline $\mathrm{Mg} /(\mathrm{Mg}+\mathrm{Fe})$ & $0 \cdot 12$ & $0 \cdot 13$ & $0 \cdot 13$ & $0 \cdot 14$ & $0 \cdot 19$ & $0 \cdot 22^{\circ}$ & $0 \cdot 23$ \\
\hline Alm & $69 \cdot 33$ & $72 \cdot 42$ & $70 \cdot 88$ & $70 \cdot 60$ & $67 \cdot 44$ & $67 \cdot 78$ & $67 \cdot 74$ \\
\hline Pyr & $9 \cdot 18$ & $10 \cdot 98$ & 13.87 & $14 \cdot 50$ & $19 \cdot 33$ & $19 \cdot 58$ & $20 \cdot 11$ \\
\hline Spe & $9 \cdot 46$ & $4 \cdot 61$ & $2 \cdot 24$ & 1.98 & $2 \cdot 19$ & 2.51 & 2.54 \\
\hline Gro & $7 \cdot 81$ & $9 \cdot 88$ & $9 \cdot 58$ & $11 \cdot 54$ & $9 \cdot 32$ & $7 \cdot 64$ & 8.89 \\
\hline Adr & $2 \cdot 82$ & $1 \cdot 40$ & $2 \cdot 29$ & 0.92 & 1.00 & 1.66 & 0.09 \\
\hline
\end{tabular}


TABLE 3 (Continued)

\begin{tabular}{|c|c|c|c|}
\hline & \multicolumn{2}{|c|}{ Sample FAY26 } & \multirow{2}{*}{$\begin{array}{l}\text { Sample FAY2S } \\
\text { Rim }\end{array}$} \\
\hline & Core & $\operatorname{Rim}$ & \\
\hline $\mathrm{SiO}_{2}$ & $36.43(0.43)$ & $37 \cdot 88(0 \cdot 26)$ & $38 \cdot 10(0 \cdot 30)$ \\
\hline $\mathrm{TiO}_{2}$ & $0.06(0.03)$ & $0.03(0.03)$ & $0.03(0.04)$ \\
\hline $\mathrm{Al}_{2} \mathrm{O}_{3}$ & $21.33(0 \cdot 25)$ & $21 \cdot 60(0 \cdot 17)$ & $21.51(0.09)$ \\
\hline $\mathrm{Cr}_{2} \mathrm{O}_{3}$ & $0.00(0.00)$ & $0.00(0.00)$ & $0.02(0.02)$ \\
\hline $\mathrm{Fe}_{2} \mathrm{O}_{3}$ & $0.73(0.47)$ & $0.26(0.15)$ & $0.20(0.07)$ \\
\hline $\mathrm{FeO}$ & $31 \cdot 14(0 \cdot 04)$ & $33 \cdot 18(0-37)$ & $30.09(0.29)$ \\
\hline $\mathrm{MnO}$ & $3.33(0.13)$ & $0.54(0.13)$ & $0.73(0.04)$ \\
\hline $\mathrm{MgO}$ & $2.22(0.09)$ & $5.84(0.42)$ & $6 \cdot 79(0 \cdot 17)$ \\
\hline $\mathrm{CaO}$ & $4 \cdot 88(0 \cdot 20)$ & $1 \cdot 17(0 \cdot 10)$ & $2 \cdot 11(0 \cdot 45)$ \\
\hline$\Sigma$ oxides & $100 \cdot 11(0 \cdot 69)$ & $100 \cdot 51(0-54)$ & $99 \cdot 55(0 \cdot 18)$ \\
\hline $\mathrm{Si}$ & 2.936 & $2 \cdot 981$ & 2.994 \\
\hline $\mathrm{Al}^{\mathrm{IV}}$ & 0.064 & 0.019 & 0.006 \\
\hline$\Sigma^{\mathrm{IV}}$ & 3.000 & 3.000 & 3.000 \\
\hline $\mathrm{Al}^{\mathrm{VI}}$ & 1.963 & 1.986 & 1.987 \\
\hline $\mathrm{Ti}$ & 0.003 & $0-002$ & 0001 \\
\hline $\mathrm{Cr}$ & 0.000 & $0-000$ & $0-001$ \\
\hline $\mathrm{Fe}^{3+}$ & 0.045 & $0 \cdot 016$ & 0.012 \\
\hline$\Sigma^{\mathrm{vl}}$ & $2 \cdot 011$ & $2 \cdot 003$ & $2 \cdot 002$ \\
\hline $\mathrm{Fe}^{2+}$ & $2 \cdot 099$ & $2 \cdot 184$ & 1.978 \\
\hline $\mathrm{Mn}$ & 0.228 & 0.036 & $0 \cdot 048$ \\
\hline $\mathrm{Mg}$ & 0.267 & 0.685 & 0.796 \\
\hline $\mathrm{Ca}$ & 0.421 & 0.099 & 0.178 \\
\hline$\Sigma^{2+}$ & $3 \cdot 014$ & $3 \cdot 004$ & 3.000 \\
\hline $\mathrm{Mg} /(\mathrm{Mg}+\mathrm{Fe})$ & $0 \cdot 11$ & $0 \cdot 24$ & $0 \cdot 29$ \\
\hline Alm & $69 \cdot 63$ & $72 \cdot 70$ & 65.94 \\
\hline Pyr & $8 \cdot 85$ & $22 \cdot 81$ & 26.52 \\
\hline Spe & $7 \cdot 55$ & $1 \cdot 19$ & $1 \cdot 61$ \\
\hline Gro & 11.58 & $2 \cdot 43$ & $5 \cdot 21$ \\
\hline Adr & 1.59 & 0.57 & 0.44 \\
\hline
\end{tabular}

$\mathrm{Fe}^{3+}$ is estimated by stoichiometry. Each analysis is an average of three to four spot analyses clustered within a small area. Values in parentheses are standard deviations. Increasing numbers denote locations of clusters within garnets from core to rim. 

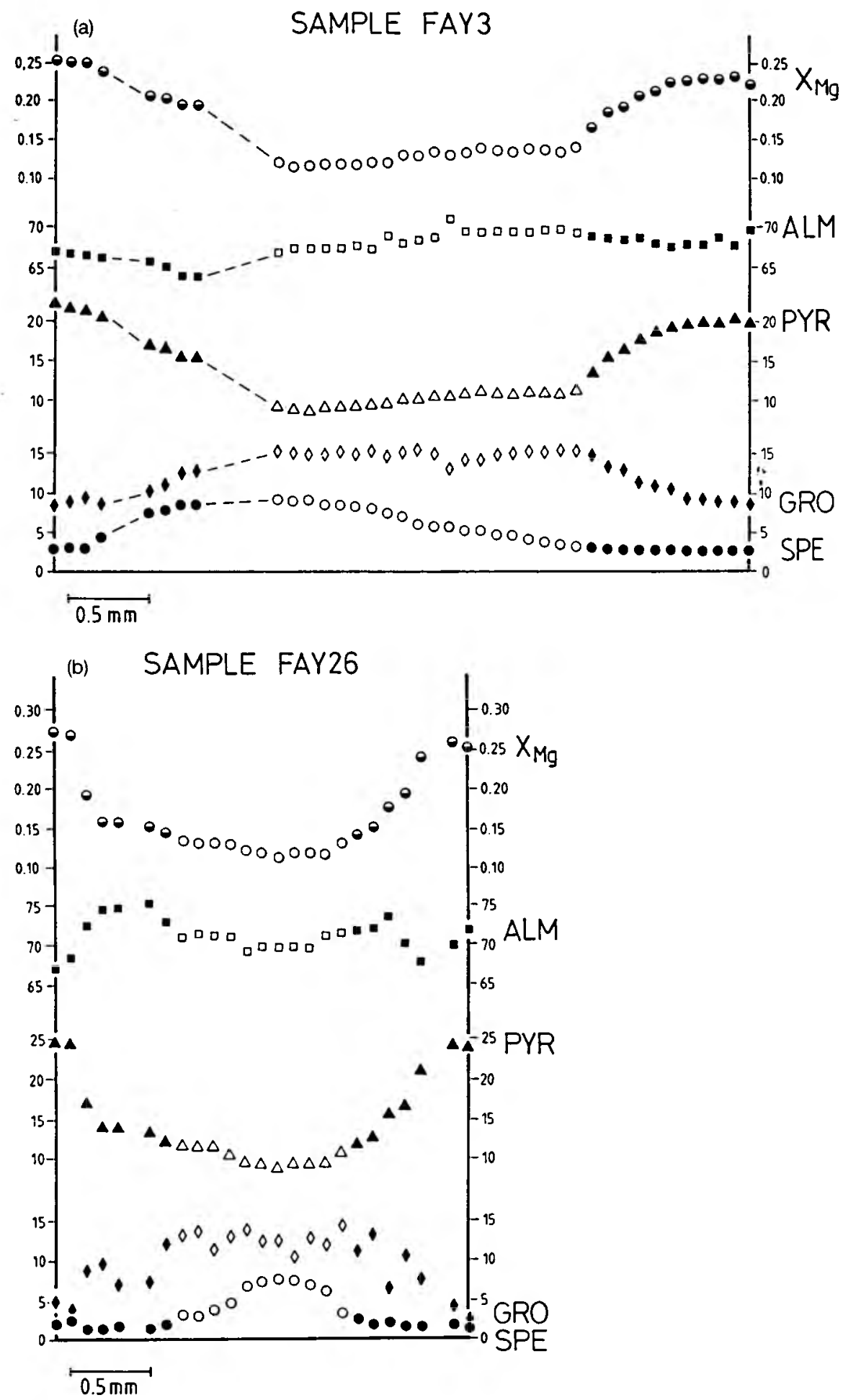

FIG. 4. Compositional zoning in garnets from samples FAY3 (a) and FAY26 (b). Open symbols represent analyses from $\mathrm{Cld}+\mathrm{St}$ inclusion-rich cores. Full symbols represent analyses from $\mathrm{Ky}$-bearing rims (FAY3) and $\mathrm{Ky}+\mathrm{Cld}$ bearing rims (FAY26). 
TABLE 4

Representative chloritoid analyses and structural formulae on a basis of 11 oxygens

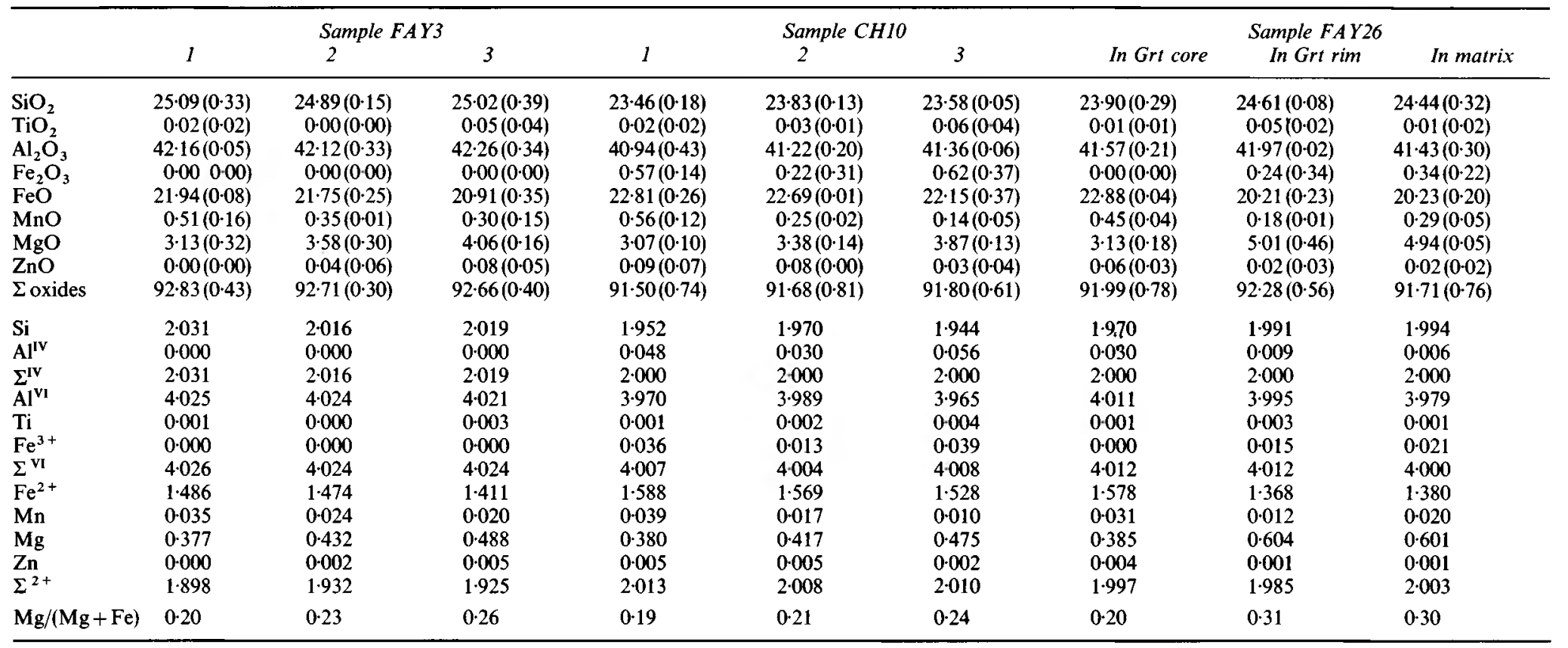

$\mathrm{Fe}^{3+}$ is estimated by stoichiometry. Each analysis is an average of three to four spot analyses clustered in a small area denoted by the same number as the enclosing garnet. Values in parentheses are standard deviations. 
TABLE 5

Representative staurolite analyses and structural formulae on a basis of 23 oxygens

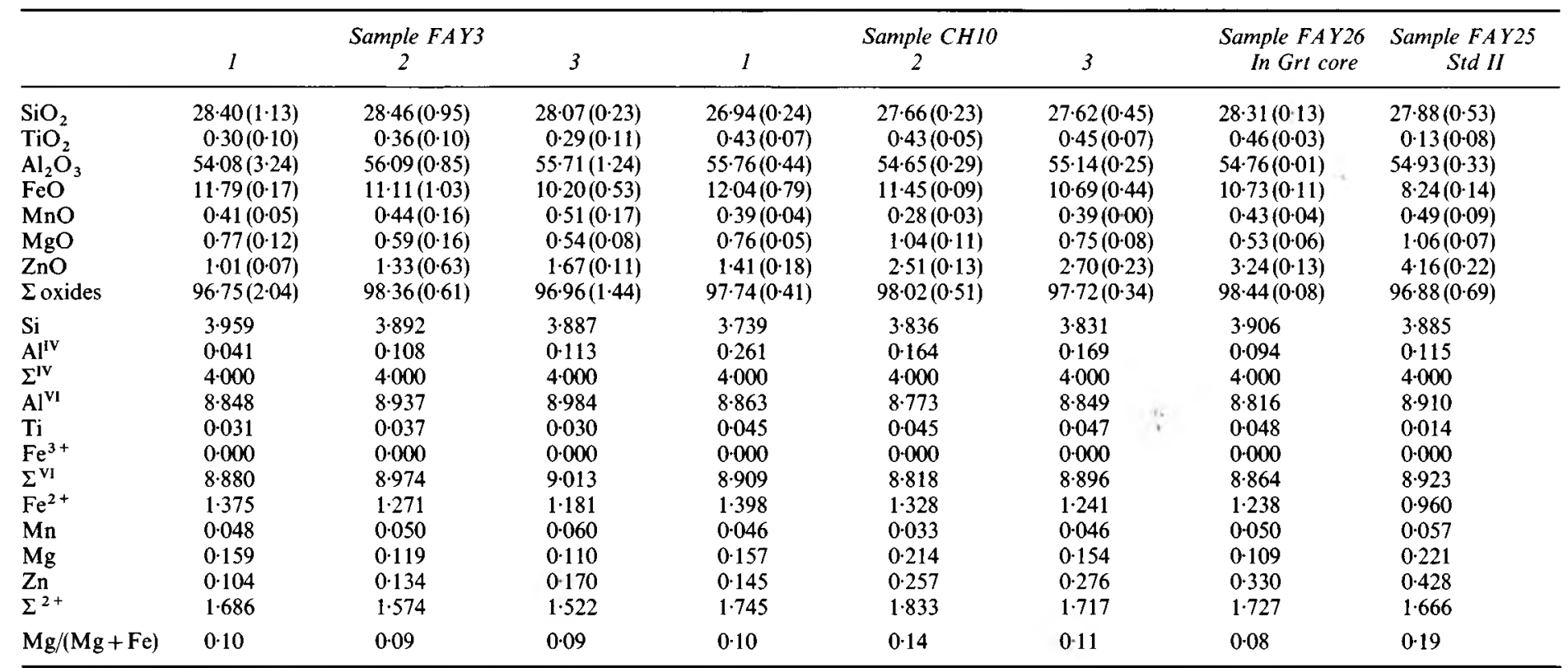

All $\mathrm{Fe}$ is calculated as $\mathrm{Fe}^{2+}$. Each analysis is an average of three to four spot analyses clustered in a small area denoted by the same number as the enclosing garnet. Values in parentheses are standard deviations. 


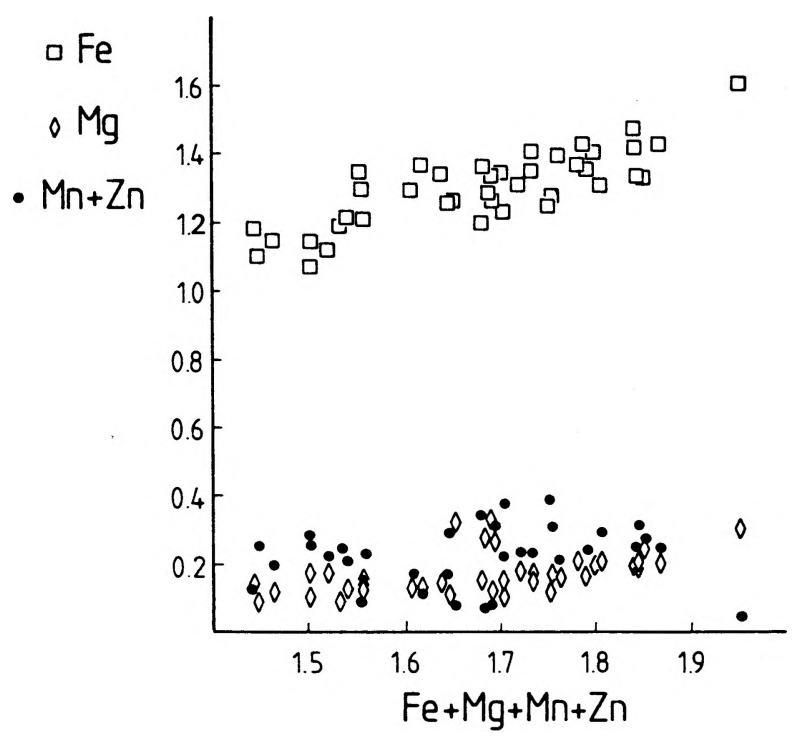

Fig. 5. $\mathrm{Fe}, \mathrm{Mg}$, and $\mathrm{Mn}+\mathrm{Zn}$ content against sum of divalent cations in staurolite inclusions from the Champtoceaux micaschists. Numbers represent cations per formula unit.

The most important feature of these staurolites is their low $X_{\mathrm{Mg}}$ value. All staurolites have lower $X_{\mathrm{Mg}}$ than coexisting garnets (see later). A comparison of our staurolite analyses with those from the Dora-Maira micaschists (Chopin, 1985) shows that the Champtoceaux staurolites have higher $\mathrm{Mn}+\mathrm{Zn}$ contents.

The analyses performed in the present study suggest the following remarks:

(1) The sum of divalent cations $\left(\mathrm{R}^{2+}\right)$ is variable and always significantly smaller than 2 . In contrast, the staurolite analyses of Holdaway et al. (1986a) have $\mathrm{R}^{2+}$ always equal to or greater than 2. The same is true for staurolite from Rangely Quadrangle (Guidotti, 1974) or Picuris Range (Holdaway, 1978). According to Holdaway et al. (1986b, fig. 1), low $\mathrm{R}^{2+}$ suggests a high $(H+F)$ content. For a mean value of $R^{2+}$ equal to $1 \cdot 70$, the suggested $(H$ $+\mathrm{F}$ ) value would be $2 \cdot 35$. The $\mathrm{MnO}$ content of analysed staurolites is always very small (less than $0.5 \mathrm{wt} \%$, i.e., 0.06 cation per formula unit).

(2) Neither $\mathrm{Mg}$ nor $\mathrm{Mn}+\mathrm{Zn}$ shows sympathetic variation with $\mathrm{R}^{2+}$, whereas $\mathrm{Fe}$ does. Thus, $\mathrm{R}^{2+}$ variations seem to be related to $\mathrm{Fe}^{2+}$ variations (Fig. 5). These observations are consistent with previous studies, suggesting an incomplete $\mathrm{Fe}-\mathrm{Mg}$ exchange in the staurolite structure (Grambling, 1983; Holdaway et al., 1986b).

(3) As shown by Griffen et al. (1982), $\mathrm{Al}^{\mathrm{VI}}$ and $\mathrm{R}^{2+}$ are strongly correlated but this is probably a consequence of the fixed $\mathrm{H}^{+}$content assumed in the calculation of structural formulae (Holdaway et al., 1986a). The latter authors suggested that $\mathrm{H}^{+}$content in staurolite is variable and they proposed a method for its estimation. This method (assuming $\mathrm{Al}+\mathrm{Si}$ $=25.53)$ has been tested, but unlikely high $\mathrm{H}^{+}$values $(>2.5)$ have been calculated as a result.

\section{White micas (Table 6)}

The maximum celadonite content of primary phengite lies around $\mathrm{Si}=3 \cdot 3$, with high $X_{\mathrm{Mg}}$ values ranging from 0.75 to $0.80 . \mathrm{Na} /(\mathrm{Na}+\mathrm{K})$ is generally low, smaller than 0.15 (Fig. 6). 
TABLE 6

Representative white mica analyses and structural formulae on a basis of 11 oxygens

\begin{tabular}{|c|c|c|c|c|c|c|}
\hline - & $\begin{array}{c}\text { Phg I } \\
13\end{array}$ & $\underset{14}{P h g ~ I}$ & $\begin{array}{c}\text { Phg II } \\
15\end{array}$ & $\begin{array}{c}\text { Phg II } \\
16\end{array}$ & $\begin{array}{c}P h g I \\
32\end{array}$ & $\begin{array}{l}P g \\
33\end{array}$ \\
\hline $\mathrm{SiO}_{2}$ & 48.81 & 48.90 & $46 \cdot 22$ & $46 \cdot 52$ & $49 \cdot 53$ & 46.99 \\
\hline $\mathrm{TiO}_{2}$ & 0.56 & 0.69 & 0.20 & $0 \cdot 23$ & 0.44 & 0.07 \\
\hline $\mathrm{Al}_{2} \mathrm{O}_{3}$ & $31 \cdot 05$ & 31.09 & $36 \cdot 70$ & 37.66 & $30 \cdot 21$ & $39 \cdot 08$ \\
\hline $\mathrm{Cr}_{2} \mathrm{O}_{3}$ & 0.00 & 0.06 & 000 & 0.06 & 000 & 000 \\
\hline $\mathrm{FeO}$ & 1.43 & 1.43 & 0.80 & 0.46 & 2.06 & 0.58 \\
\hline $\mathrm{MnO}$ & 0.00 & 000 & $0-00$ & 0.06 & 000 & 0.01 \\
\hline $\mathrm{MgO}$ & 2.62 & $2 \cdot 40$ & 0.85 & 0.28 & $2 \cdot 47$ & 0.22 \\
\hline $\mathrm{CaO}$ & 0.00 & 000 & $0-00$ & 000 & 000 & 0.24 \\
\hline $\mathrm{Na}_{2} \mathrm{O}$ & 0.72 & 0.80 & 1.47 & 1.75 & 0.72 & $6 \cdot 54$ \\
\hline $\mathrm{K}_{2} \mathrm{O}$ & 8.76 & 8.98 & 8.39 & $8 \cdot 17$ & $10 \cdot 25$ & 1.22 \\
\hline$\Sigma$ oxides & 93.95 & $94 \cdot 35$ & 94.63 & $95 \cdot 19$ & 95.68 & 94.95 \\
\hline $\mathrm{Si}$ & $3 \cdot 259$ & 3.257 & 3.056 & 3.049 & $3 \cdot 286$ & 3.016 \\
\hline $\mathrm{Al}^{\mathrm{IV}}$ & 0.741 & 0.743 & 0.944 & 0.951 & 0.714 & 0.984 \\
\hline$\Sigma^{\mathrm{IV}}$ & 4000 & 4.000 & 4000 & $4 \cdot 000$ & 4000 & 4.000 \\
\hline $\mathrm{Al}^{\mathrm{VI}}$ & $1 \cdot 703$ & 1.698 & 1.917 & 1.960 & 1.649 & 1.973 \\
\hline $\mathrm{Ti}$ & 0.028 & 0.035 & 0.010 & 0.011 & 0.022 & $0-003$ \\
\hline $\mathrm{Cr}$ & $0-000$ & $0-003$ & $0-000$ & $0-003$ & $0-000$ & 0.000 \\
\hline $\mathrm{Fe}^{2+}$ & 0.080 & 0.080 & $0-044$ & 0.025 & $0 \cdot 114$ & 0.031 \\
\hline Mn & $0-000$ & $0-000$ & $0-000$ & 0.003 & $0-000$ & $0-001$ \\
\hline $\mathrm{Mg}$ & 0.261 & 0.238 & 0.084 & 0.027 & 0.244 & 0.021 \\
\hline$\Sigma^{\mathrm{vi}}$ & 2.072 & $2 \cdot 054$ & 2.055 & 2.029 & 2.029 & 2.029 \\
\hline $\mathrm{Ca}$ & $0-000$ & $0-000$ & $0-000$ & $0-000$ & $0-000$ & 0.017 \\
\hline $\mathrm{Na}$ & 0.093 & $0 \cdot 103$ & $0 \cdot 188$ & 0.222 & 0.093 & 0.814 \\
\hline $\mathbf{K}$ & 0.746 & 0.763 & 0.708 & 0.683 & 0.868 & $0 \cdot 100$ \\
\hline$\Sigma$ int & 0.839 & 0.866 & 0.896 & 0.905 & 0.961 & 0.931 \\
\hline
\end{tabular}

All $\mathrm{Fe}$ is calculated as $\mathrm{Fe}^{2+}$.

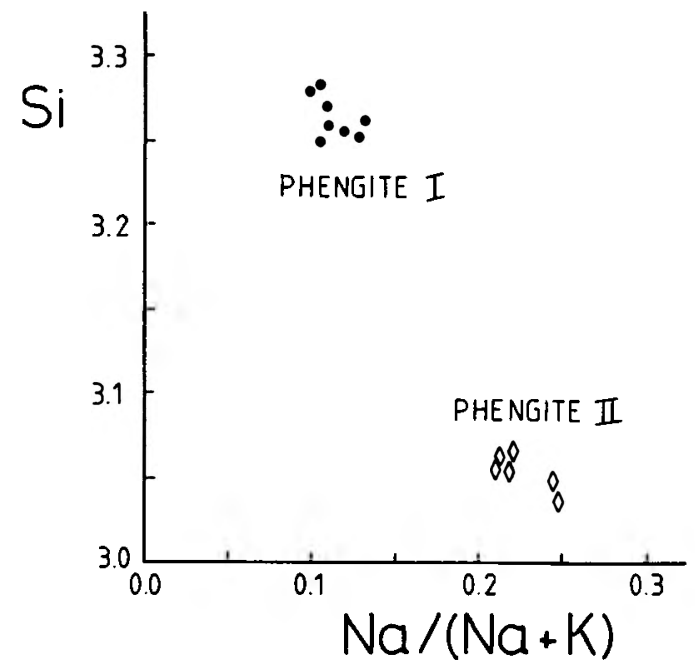

FIG. 6. Si content against $\mathrm{Na} /(\mathrm{Na}+\mathrm{K})$ ratio in phengites from the Champtoceaux micaschists (sample $\mathrm{Cx} 12$ ). 
Secondary phengites have a lower celadonite content $(\mathrm{Si}=3 \cdot 05-3 \cdot 10)$ than primary phengites, but a higher $\mathrm{Na} /(\mathrm{Na}+\mathrm{K})$ ratio $(0 \cdot 2-0 \cdot 25)$ (Fig. 6).

Paragonite has a low $\mathrm{Ca}$ content $[\mathrm{Ca} /(\mathrm{Na}+\mathrm{K}+\mathrm{Ca})=0.08]$ and can have a $\mathrm{K} /(\mathrm{Na}+\mathrm{K}$ $+\mathrm{Ca})$ ratio ranging up to $0 \cdot 10$.

\section{Kyanite}

Kyanite is always nearly pure, with only trace amounts of metallic cations.

\section{Chlorite}

Chlorite compositions are relatively heterogeneous ( $X_{\mathrm{Mg}}$ values range from 0.4 to $0 \cdot 6$ ). Variations occur in each sample and between adjacent grains. It has not been possible to distinguish primary and secondary chlorites.

\section{Plagioclase}

Plagioclase composition varies with the textural position of the grains; for example, plagioclases from sample FAY26 are purely albitic in the matrix but unzoned grains occurring in cracks in garnet contain around $15 \mathrm{~mol} \% \mathrm{An}$.

\section{PHASE RELATIONS OF STAUROLITE IN ECLOGITIC METAPELITES}

\section{Phase relations in the Champtoceaux micaschists}

The Champtoceaux micaschists can be studied in the KFMASH system. MnO and $\mathrm{CaO}$ are considered as negligible components because they are strongly partitioned into garnets. Assuming that $\mathrm{H}_{2} \mathrm{O}$ is in excess, and taking into account the presence of quartz and

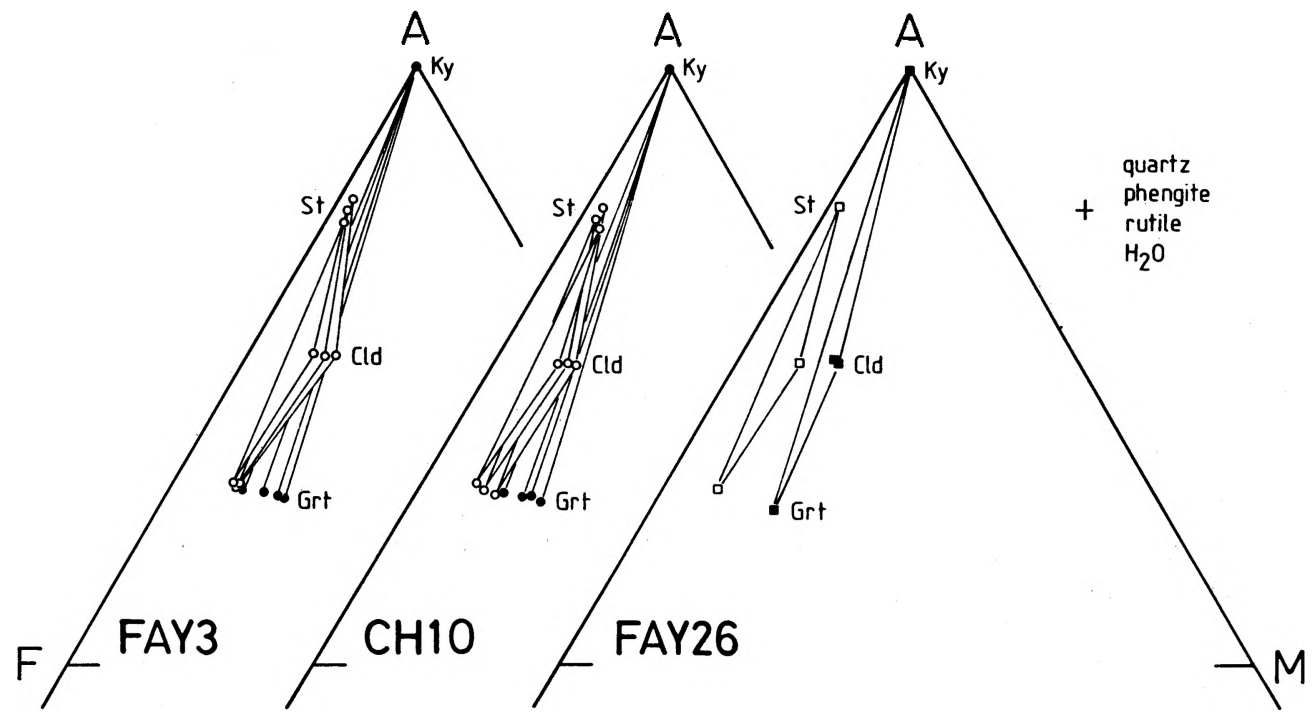

FIG. 7. $\mathrm{Al}_{2} \mathrm{O}_{3}-\mathrm{FeO}-\mathrm{MgO}$ projection from quartz, rutile and $\mathrm{H}_{2} \mathrm{O}$ of coexisting minerals in the Champtoceaux micaschists. Open symbols represent garnet inclusions and coexisting garnets. Filled symbols represent garnet rims and matrix phases. Projected mineral compositions are reported in Tables 3-5. 
phengite, the AFM projection can be used to show phase relations in the Champtoceaux micaschists (Fig. 7). Textural observations and chemical analyses show that the primary assemblage represents a state of partial equilibrium (Loomis, 1983): chloritoid and staurolite are in equilibrium with garnet cores, but are not in equilibrium with garnet rims and matrix phases. The local development and chemical heterogeneity of secondary phases suggests that only local equilibrium was achieved during the retrograde history. Thus, their use for reconstructing the $P-T$ path of the Champtoceaux micaschists will be more uncertain than the use of relict prograde phases included within garnet cores.

\section{Primary assemblages}

In the studied samples, the matrix equilibrium assemblage is garnet rim + kyanite ( + phengite + quartz + rutile). Inclusions of staurolite \pm chloritoid in garnet cores are used to define prograde assemblages.

All samples show a striking evolution from a garnet-chloritoid-staurolite assemblage (garnet cores) towards a garnet-kyanite association (garnet rims + matrix phases) (Fig. 7). Coexisting garnet-staurolite-kyanite was never observed, but garnet-chloritoid-kyanite was stable in sample FAY26, where chloritoid grains are present in the matrix. During the final stages of the metamorphic history, matrix chloritoid reacts with matrix phases and is now enclosed in a phengitic aggregate (Fig. 3), or separated from garnet and kyanite by chlorite sheaves.

Staurolite is always more Fe-rich and chloritoid more Mg-rich than the coexisting garnet (Fig. 7). In other words, the Fe-Mg partitioning between AFM phases is, according to an increasing $X_{\mathrm{Mg}}$ ratio: $\mathrm{St}<\mathrm{Grt}<\mathrm{Cld}$.

Garnet shows, from core to rim, an increasing $X_{\mathrm{Mg}}$ ratio. Chemical gradients are relatively smooth in the chloritoid + staurolite inclusion-rich cores but strongly increase in garnet rims. Chloritoid inclusions evolve towards more $\mathrm{Mg}$-rich compositions from the inner to the outer zone of garnet cores. In addition, chloritoid inclusions in staurolite-free garnet rims and matrix chloritoid grains have similar compositions, which are much more magnesian than the last chloritoid inclusions coexisting with staurolite (Fig. 7). The chemical evolution of staurolite could not be precisely constrained, because of the narrow $\mathrm{Fe}-\mathrm{Mg}$ substitution range and chemical heterogeneities within single grains.

\section{Secondary assemblages}

Secondary chlorite-staurolite associations are observed only in sample FAY25: staurolite II presents a higher $X_{\mathrm{Mg}}$ (of about $0 \cdot 19$ ) than staurolite I, but chlorite compositions are variable $\left(X_{\mathrm{Mg}}=0.40-0.60\right)$. The chemical heterogeneity of plagioclase grains depends on local equilibria. Biotite is not observed.

\section{$F e-M g$ partitioning between garnet and staurolite}

A literature survey shows that staurolites have a higher $X_{\mathrm{Mg}}$ than coexisting garnets in low- to medium-pressure metapelites, but that the reverse is true in high-pressure metapelites. Numerous studies are available in low- to medium-pressure metapelites (e.g., Albee, 1972; Guidotti, 1974; Holdaway, 1978; Yardley et al., 1980; Pigage \& Greenwood, 1982; Grambling, 1983; Lang \& Rice, 1985; Gibson \& Speer, 1986; Klaper \& BucherNurminen, 1987; Holdaway et al., 1988). In contrast, only five occurrences are reported in high-pressure metapelites (see Introduction).

Figure 8 presents a compilation of $\mathrm{Fe} / \mathrm{Mg}$ values for coexisting garnet and staurolite. From the numerous papers on low- to medium-pressure micaschists, we extract analyses 

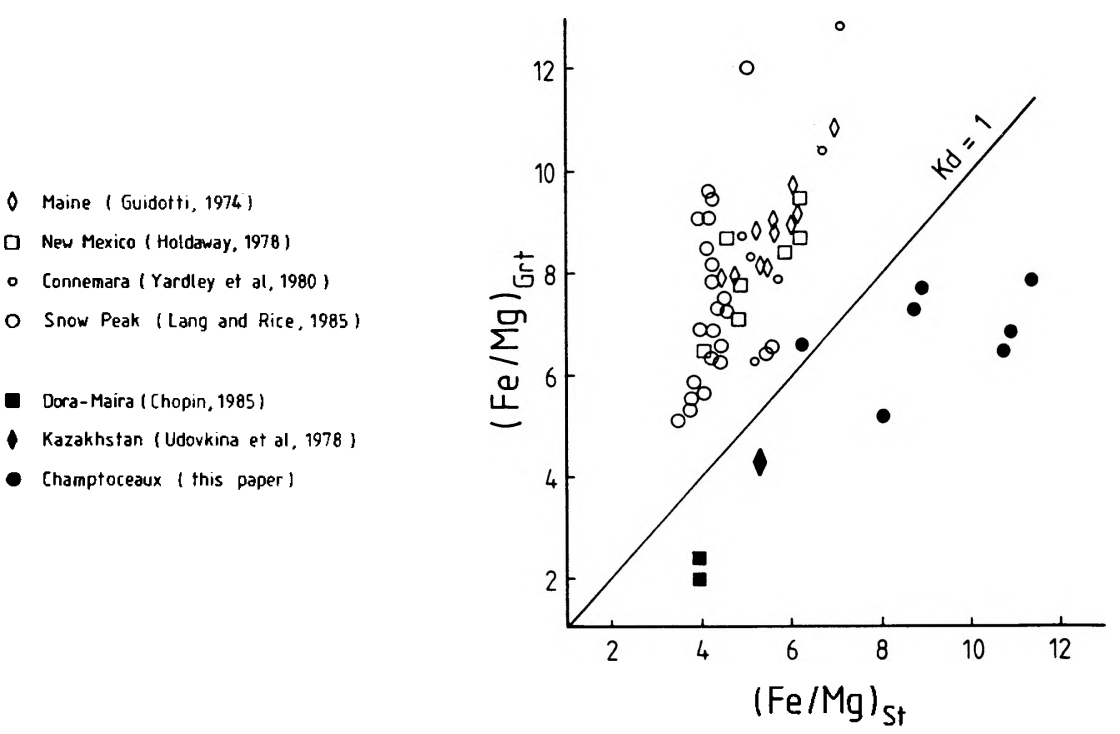

Fig. 8. (Fe/Mg) in staurolite vs. (Fe/Mg) in coexisting garnet. Data for the Champtoceaux micaschists are taken from Tables 3 and 5 .

from coexisting garnets and staurolites which have crystallized at almost fixed $P-T$ (Holdaway, 1978; Lang \& Rice, 1985) or along a $P-T$ 'gradient' (Guidotti, 1974; Yardley et al., 1980). All available data for high-pressure metapelites have been reported (i.e., from Kazakhstan and Dora-Maira), but only mean analyses were used for the Champtoceaux micaschists. Figure 8 clearly shows that $K_{d}$ values are greater than 1 in medium-pressure metapelites but less than 1 in high-pressure metapelites. It thus demonstrates that a reversal of $\mathrm{Fe}-\mathrm{Mg}$ partitioning occurs between garnet and staurolite.

Such a reversal has already been described between chloritoid and staurolite by Grambling (1983). He paid particular attention to possible misinterpretations of chemical data. According to him, apparent $\mathrm{Fe}-\mathrm{Mg}$ reversal could result from (1) disequilibrium relations between garnet and staurolite, or (2) perturbations of the $\mathrm{Fe}-\mathrm{Mg}$ exchange caused by minor elements, notably $\mathrm{Fe}^{3+}, \mathrm{Mn}$, or $\mathrm{Zn}$. The presence of graphite + rutile and the lack

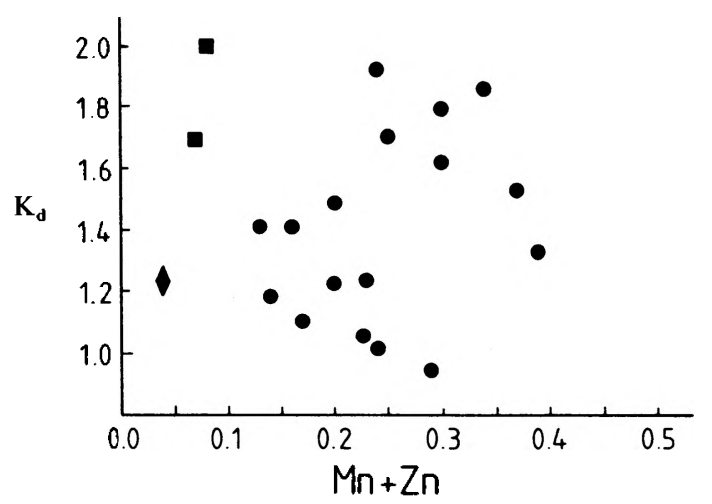

FIG. 9. Plot of $K_{d}$ vs. minor elements in staurolites. Same symbols as for Fig. 8. We used all available garnet-staurolite pairs from the Champtoceaux micaschists for sake of complete representation. 
of ilmenite, magnetite, or haematite (Table 2) suggest a low oxygen fugacity. Very low $\mathrm{Fe}^{3+}$ estimates in garnet and chloritoid are consistent with the preceding statement. $\mathrm{MnO}$, which essentially enters into garnet, is negligible in staurolite. $\mathrm{ZnO}$ is preferentially partitioned into staurolite, but there is no correlation between $K_{d}$ value and $\mathrm{Zn}+\mathrm{Mn}$ content (Fig. 9). Disequilibrium between garnet and staurolite cannot be totally excluded but no evidence for it could be found.

In conclusion, available data strongly favour the existence of an inverse $\mathrm{Fe}-\mathrm{Mg}$ partitioning between garnet and staurolite in high-pressure metapelites, possibly as a result of the non-ideal behaviour of the Fe-Mg solid solution in staurolite suggested by Holdaway et al. (1988). Preliminary experimental investigations of $\mathrm{Fe}-\mathrm{Mg}$ partitioning between garnet and staurolite (Rice, 1985) also show the occurrence of such a reversal.

\section{Topological consequences of the $\mathrm{Fe}-\mathrm{Mg}$ reversal}

Most authors have analysed phase relations in metapelites using the KFMASH multisystem (e.g., Albee, 1972; Harte \& Hudson, 1979; Koons \& Thompson, 1985; Vuichard \& Ballèvre, 1988). In fact, most metapelites can be rigorously described using the AFM projection (e.g., Thompson, 1957; Greenwood, 1975).

This paper focuses on phase relations between garnet, staurolite, chloritoid, chlorite, and kyanite (with excess quartz, muscovite, and $\mathrm{H}_{2} \mathrm{O}$ ) in high-pressure metapelites. We are thus concerned only with reactions around the [Crd, Bt] KFMASH invariant point of Harte \& Hudson (1979), which is equivalent to the [Bt, Tlc] invariant point of Vuichard \& Ballèvre (1988). These authors assumed a normal $\mathrm{Fe}-\mathrm{Mg}$ partitioning between AFM phases. If a reversal of $\mathrm{Fe}-\mathrm{Mg}$ partitioning between garnet and staurolite occurs the three terminal staurolite-out reactions:

$$
\begin{gathered}
\mathrm{St}=\mathrm{Grt}+\mathrm{Cld}+\mathrm{Ky} \\
\mathrm{St}=\mathrm{Grt}+\mathrm{Chl}+\mathrm{Ky} \\
\mathrm{St}=\mathrm{Grt}+\mathrm{Bt}+\mathrm{Ky}
\end{gathered}
$$

are replaced by the non-terminal reactions:

$$
\begin{gathered}
S t+C l d=G r t+K y \\
S t+C h l=G r t+K y \\
S t+B t=G r t+K y .
\end{gathered}
$$

Therefore, the staurolite stability field is extended toward higher pressures and temperatures than in the case of a normal partitioning.

We will now consider in some detail what occurs around the KFMASH invariant point [Bt, Tlc] which is critical for the understanding of phase relations in high-pressure metapelites. Figure 10 shows the topology of univariant KFMASH reactions around the [Bt, Tlc] invariant point with normal $\mathrm{Fe}-\mathrm{Mg}$ partitioning (Fig. 10a) and with inverse partitioning (Fig. 10b). If $X_{\mathrm{Mg}}^{\mathrm{St}}=X_{\mathrm{Mg}}^{\mathrm{Grt}}$, the degenerate reaction

$$
\mathrm{St}=\mathrm{Grt}+\mathrm{Ky}
$$

will be coincident with

$$
\mathrm{Fe}_{-} \mathrm{St}=\mathrm{Alm}+\mathrm{Ky}
$$

eliminating the (Bt, Chl, Tlc) and (Bt, Cld, Tlc) KFMASH reactions by degeneracy. Thus 

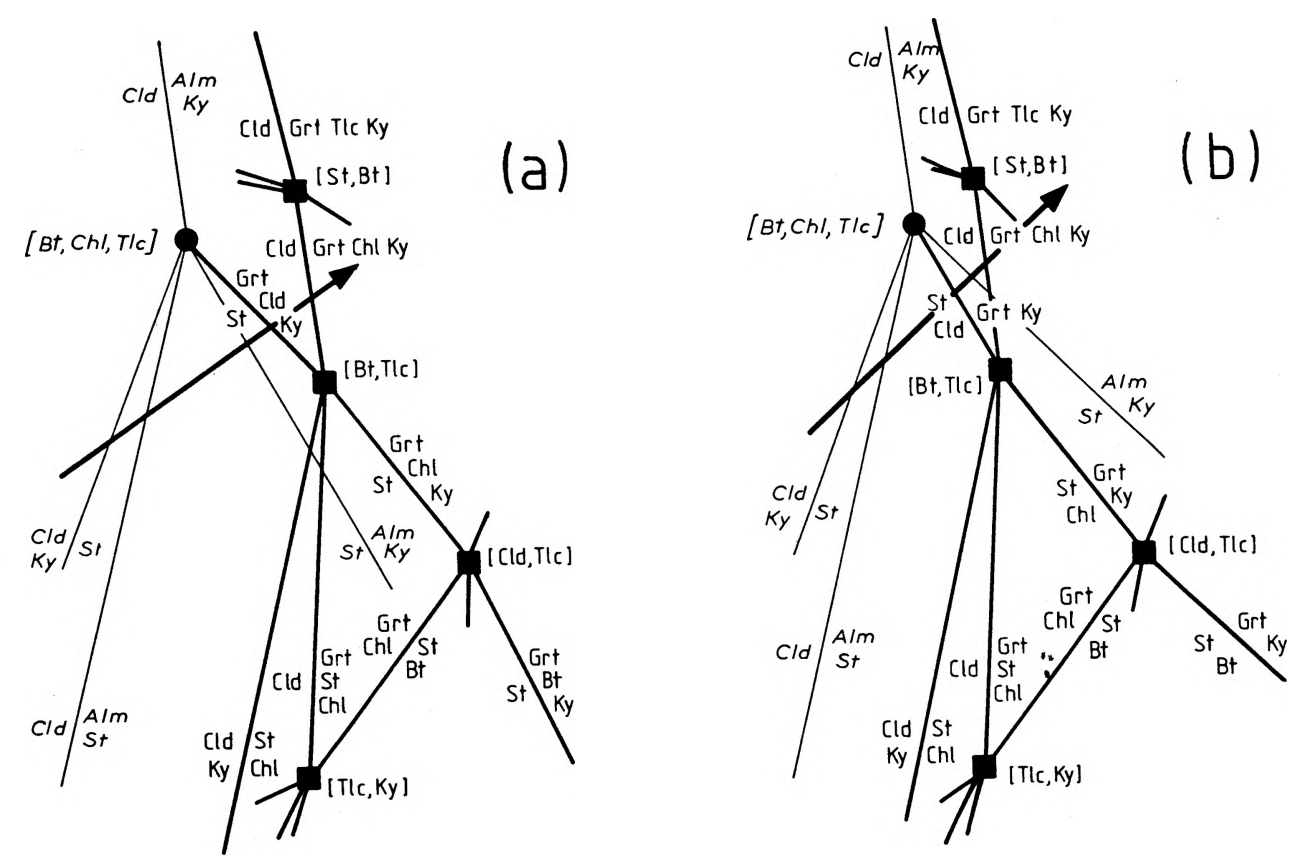

FIG. 10. Phase relations of garnet, chloritoid, staurolite, biotite, and chlorite in the KFMASH multisystem for normal, i.e., Barrovian (a) and reverse (b) Fe-Mg partitioning between garnet and staurolite. Squares and heavy lines refer to invariant points and univariant reactions in the KFMASH system. Full circles and light lines refer to invariant points and univariant reactions in the FASH system, respectively.

Fig. 10a will change to Fig. 10b at least in part of the $P-T$ field, by $\mathrm{Fe}_{-} \mathrm{Mg}$ changes along the $(\mathrm{Bt}, \mathrm{Chl}, \mathrm{Tlc})$ or $(\mathrm{Bt}, \mathrm{Cld}, \mathrm{Tlc})$ reactions. The inversion may occur at pressures between the invariant points $[\mathrm{Bt}, \mathrm{Tlc}]$ and $[\mathrm{Bt}, \mathrm{Chl}, \mathrm{Tlc}]$ or $[\mathrm{Bt}, \mathrm{Tlc}]$ and $[\mathrm{Cld}, \mathrm{Tlc}]$. Another way to relate Fig. 10a and Fig. $10 \mathrm{~b}$ is to consider that each Schreinemakers net represents a projection in the $P-T$ plane of univariant reaction surfaces in a three-dimensional space. The third intensive parameter is not identified at present but could be the oxygen fugacity (see, for example, Hensen, 1986) or the hydrogen content of staurolite.

Figure 11 shows the predicted Fe-Mg evolution of AFM phases along a particular $P-T$ path (arrows on Fig. 10) for aluminous bulk-rock compositions. We also represent the two types of $\mathrm{Fe}-\mathrm{Mg}$ partitioning. Two main consequences are to be stressed:

(1) An $\mathrm{Fe}-\mathrm{Mg}$ reversal does not enable a strong $\mathrm{Mg}$-enrichment in staurolite, contrary to the assessment of Vuichard \& Ballèvre (1988). In fact, magnesian staurolites coexisting with garnet have been reported only from quartz-free metabasites (Smith, 1984; Ward; 1984; Enami \& Zang, 1988) or silica-deficient peraluminous rocks (Grew \& Sandiford, 1984; Schreyer et al., 1984). The last two studies are concerned with non-metapelitic rocks where $\mathrm{SiO}_{2}$ is not in excess. In consequence, the hypotheses required for AFM projection are not fulfilled. Moreover, Schreyer et al. (1984) stated that such magnesian staurolites cannot be stable with quartz, as with pure Mg-staurolite (Schreyer, 1968).

(2) Garnet-kyanite is a critical assemblage in eclogitic metapelites: its occurrence extends towards more magnesian compositions with increasing $P-T$ conditions. Thus, most highpressure metapelites would appear as monotonous garnet-kyanite micaschists. 


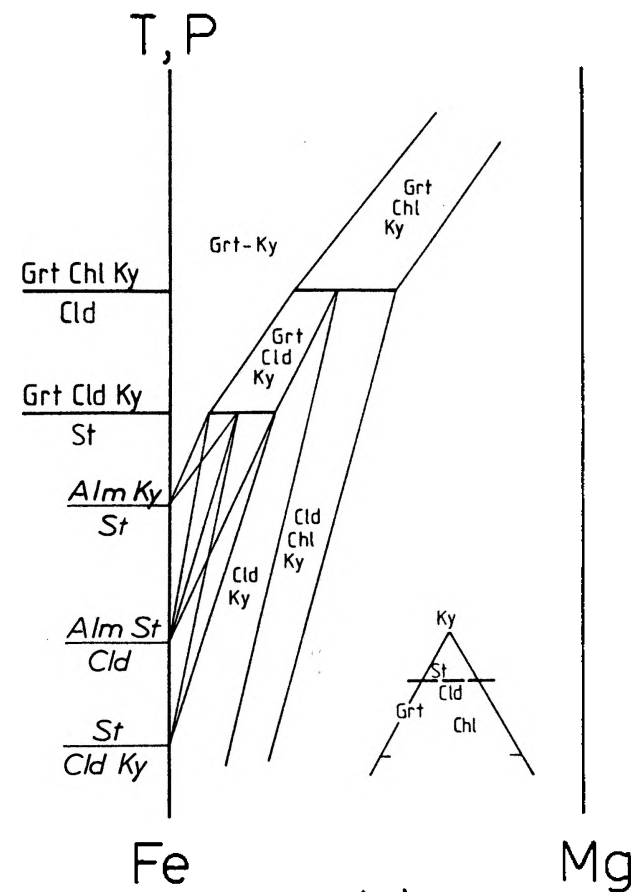

(a)

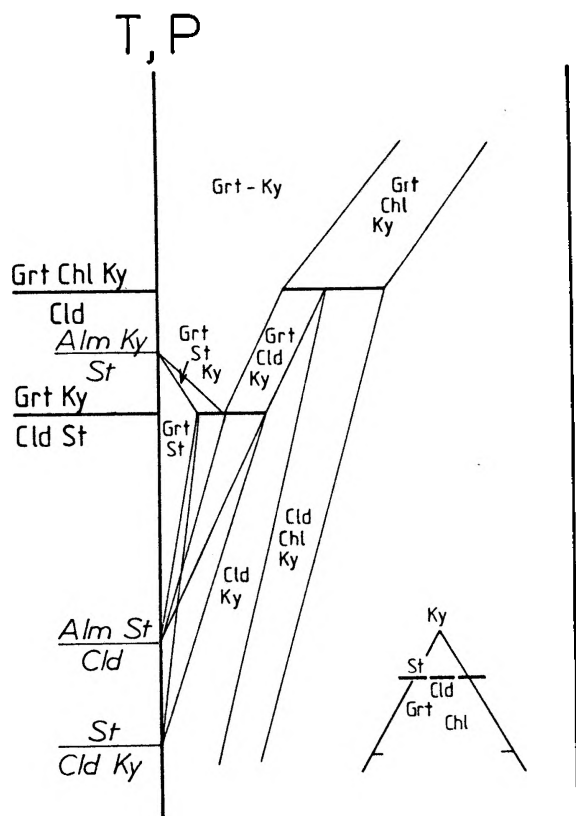

(b)

FIG. 11. Schematic pseudo-binary loops for aluminous, ferrous to intermediate bulk-rock compositions. The diagrams depict the evolution of mineral compositions and assemblages along a $P-T$ gradient (arrows on Fig. 10) for a normal (a) and a reverse (b) $\mathrm{Fe}-\mathrm{Mg}$ partitioning between garnet and staurolite.

The main limitation of the above approach is that pseudo-binary loops do not take into account substitutions other than Fe-Mg and non-ideal mixing behaviour in AFM phases.

\section{A model reaction history for the Champtoceaux micaschists}

\section{Prograde reaction history}

As a whole, the observed parageneses in the Champtoceaux micaschists and their predicted evolution in the KFMASH system are consistent. Some points deserve further comment.

(1) Reaction (4) describes the actual reaction path in the studied micaschists. The garnet-chloritoid-staurolite assemblage is present in the garnet cores of all studied samples (Fig. 7). Garnet-staurolite-kyanite and garnet-chloritoid-kyanite assemblages are stable on the high-pressure side of reaction (4) but only garnet chloritoid-kyanite is known at present in the Champtoceaux micaschists.

(2) Chlorite could result from the reaction

$$
\mathrm{Cld}=\mathrm{Grt}+\mathrm{Chl}+\mathrm{Ky}
$$

but textural evidence in favour of this is not conclusive. If this reaction actually occurred, chlorite was probably re-equilibrated during the retrograde history.

(3) Pseudo-binary loops (Fig. 11b) are compatible with the observed chemical evolution of garnet and chloritoid, in particular with the strong $\mathrm{Mg}$-enrichment after reaction (4) 
occurred. The chemical evolution of staurolite inclusions within garnet cores would tend to a slight Mg-enrichment (Fig. 11b). This is not detectable in our analyses, possibly because of (a) analytical uncertainties and (b) complex solid solution behaviour of staurolite. It should be noted that the evolution from chloritoid-staurolite-garnet to garnet-chloritoid-kyanite assemblages can occur in both $\mathrm{Fe}-\mathrm{Mg}$ partitioning schemes. Only the evolution from chloritoid-staurolite-garnet to garnet-staurolite-kyanite assemblages would be diagnostic of an inverse partitioning.

In summary, the Champtoceaux micaschists preserve part of a prograde $P-T$ path in garnet inclusion-rich cores, whereas matrix phases (kyanite and phengite) and garnet rims represent the 'peak' metamorphic conditions.

\section{Retrograde reaction history}

Reaction (5) could be used to describe the retrograde growth of chlorite + staurolite II assemblage in sample FAY25 if we assume that staurolite II, chlorite, and garnet are in equilibrium. In addition, the lack of biotite in garnet-bearing micaschists suggests that the reaction

$$
\mathrm{Grt}+\mathrm{Chl}=\mathrm{St}+\mathrm{Bt}
$$

never takes place.

\section{$P-T$ CONDITIONS OF THE ECLOGITE-FACIES METAMORPHISM}

Even if observed phase relations in the Champtoceaux micaschists clearly show that they are true eclogite-facies parageneses, it would be highly desirable to determine the $P-T$ conditions of this metamorphism. Few geothermobarometers are available for highpressure metapelites, but independent estimates in eclogite-facies metabasites and quartzites can be used for reference. Quantitative evaluation of the $P-T$ history of the studied rocks is very difficult, but the shape of the prograde $P-T$ path can be qualitatively constrained.

\section{Geothermobarometry}

Classical geobarometers are lacking in the studied micaschists, for example, garnet-plagioclase-kyanite-quartz (Newton \& Haselton, 1981). The observed associations can only be used to infer minimum or maximum pressures. In the absence of biotite and $\mathrm{K}$ feldspar, the $\mathrm{Si}^{4+}$ content of primary phengites (Massone \& Schreyer, 1987) gives minimum pressures of the order of $10 \mathrm{~kb}$ at temperatures ranging from 600 to $700^{\circ} \mathrm{C}$. In the absence of ilmenite, the GRAIL equilibrium (Bohlen et al., 1983) gives minimum pressures around $8 \mathrm{~kb}$. This equilibrium unfortunately cannot be applied to garnet cores where an aluminosilicate phase is generally lacking and coexisting rutile and ilmenite are not found (see below). In the absence of sodic pyroxene, the paragonite + kyanite assemblage gives a maximum pressure at around $25 \mathrm{~kb}$ at $700^{\circ} \mathrm{C}$ [assuming $a\left(\mathrm{H}_{2} \mathrm{O}\right)=1$ ] (Holland, 1979).

Most geothermometers are based on $\mathrm{Fe}-\mathrm{Mg}$ exchange reactions between coexisting ferromagnesian minerals, notably garnet-biotite (Ferry \& Spear, 1978) and garnet-phengite (Green \& Hellman, 1982). Given that biotite is lacking, temperatures can only be calculated using the garnet-phengite geothermometer. The use of phengite analyses with all $\mathrm{Fe}$ as $\mathrm{Fe}^{2+}$ yields unreasonably high temperature estimates ranging from 800 to $850^{\circ} \mathrm{C}$ at $20 \mathrm{~kb}$ pressure. In consequence, the $\mathrm{Fe}^{3+}$ content of phengites has been calculated assuming that $\mathrm{Mg}+\mathrm{Fe}^{2+}=\mathrm{Si}-3$. In some cases, this leads to recalculation of all $\mathrm{Fe}$ as $\mathrm{Fe}^{3+}$. Such analyses have been rejected from further calculations. Using the calibration of Green \& 
TABLE 7

Garnet-phengite thermometry in the Champtoceaux micaschists

\begin{tabular}{|c|c|c|c|c|c|c|}
\hline & \multicolumn{3}{|c|}{ Sample FAY3 } & \multicolumn{3}{|c|}{ Sample FAY 26} \\
\hline \multicolumn{7}{|l|}{ Grt } \\
\hline $\mathrm{Ca}$ & $0 \cdot 295$ & 0.272 & 0.282 & 0.099 & 0.274 & $0 \cdot 176$ \\
\hline $\mathrm{Fe}$ & $2 \cdot 025$ & $2 \cdot 038$ & $2 \cdot 026$ & $2 \cdot 207$ & $2 \cdot 049$ & 2.099 \\
\hline $\mathbf{M g}$ & 0.596 & 0.594 & 0.609 & 0.654 & 0.705 & $0 \cdot 710$ \\
\hline $\mathrm{Fe} / \mathrm{Mg}$ & $3 \cdot 40$ & 3.43 & $3 \cdot 33$ & $3 \cdot 37$ & $2 \cdot 91$ & $2 \cdot 96$ \\
\hline \multicolumn{7}{|l|}{ Phg } \\
\hline $\mathrm{Fe}$ & 0.071 & $0 \cdot 071$ & 0.071 & 0.072 & 0.067 & $0 \cdot 064$ \\
\hline $\mathrm{Mg}$ & 0.210 & $0 \cdot 210$ & $0 \cdot 210$ & $0 \cdot 254$ & $0 \cdot 254$ & 0.224 \\
\hline $\mathrm{Fe} / \mathrm{Mg}$ & $0 \cdot 34$ & $0 \cdot 34$ & $0 \cdot 34$ & $0 \cdot 28$ & $0 \cdot 26$ & 0.29 \\
\hline$K_{\mathrm{d}}$ & $10-05$ & $10 \cdot 15$ & $9 \cdot 84$ & 11.90 & 11.02 & $10 \cdot 35$ \\
\hline \multicolumn{7}{|c|}{ Temperatures $\left({ }^{\circ} \mathrm{C}\right)$} \\
\hline$P=15 \mathrm{~kb}$ & 643 & 642 & 646 & 621 & 631 & 639 \\
\hline$P=20 \mathrm{~kb}$ & 670 & 669 & 673 & 647 & 657 & 666 \\
\hline
\end{tabular}

Sample FAYGB

\begin{tabular}{|c|c|c|c|c|c|c|c|}
\hline Grt & 5 & 11 & 13 & 29 & 31 & 33 & 39 \\
\hline $\mathrm{Ca}$ & $0 \cdot 441$ & 0.309 & $0 \cdot 304$ & $0 \cdot 311$ & 0.353 & 0.329 & 0.448 \\
\hline $\mathrm{Fe}$ & $1 \cdot 784$ & 1.839 & 1.871 & 1.869 & 1.834 & 1.880 & 1.837 \\
\hline $\mathrm{Mg}$ & 0.683 & 0.754 & 0.741 & 0.752 & 0.760 & 0.748 & 0.498 \\
\hline $\mathrm{Fe} / \mathrm{Mg}$ & $2 \cdot 61$ & $2 \cdot 44$ & $2 \cdot 52$ & $2 \cdot 49$ & $2 \cdot 41$ & $2 \cdot 51$ & 3.69 \\
\hline Phg & 4 & 10 & 12 & 28 & 30 & 32 & 37 \\
\hline $\mathrm{Fe}$ & 0044 & $0-058$ & 0.060 & 0.077 & $0 \cdot 109$ & 0.055 & $0 \cdot 102$ \\
\hline $\mathrm{Mg}$ & 0.210 & 0.236 & $0 \cdot 273$ & $0 \cdot 243$ & 0.263 & 0.249 & 0.288 \\
\hline $\mathrm{Fe} / \mathrm{Mg}$ & 0.21 & $0 \cdot 25$ & 0.22 & 0.32 & 0.41 & 0.22 & $0 \cdot 35$ \\
\hline$K_{\mathrm{d}}$ & $12 \cdot 47$ & $9 \cdot 92$ & 11.49 & 7.84 & $5 \cdot 82$ & $11 \cdot 38$ & $10 \cdot 42$ \\
\hline \multicolumn{8}{|c|}{ Temperatures $\left({ }^{\circ} \mathrm{C}\right)$} \\
\hline $\begin{array}{l}P=15 \mathrm{~kb} \\
P=20 \mathrm{~kb}\end{array}$ & $\begin{array}{l}621 \\
647\end{array}$ & $\begin{array}{l}645 \\
672\end{array}$ & $\begin{array}{l}626 \\
652\end{array}$ & $\begin{array}{l}677 \\
705\end{array}$ & $\begin{array}{l}624 \\
650\end{array}$ & $\begin{array}{l}627 \\
653\end{array}$ & $\begin{array}{l}639 \\
665\end{array}$ \\
\hline
\end{tabular}

Hellman (1982) (for low Ca and low Mg systems) with garnet rims and primary phengite compositions, temperatures ranging from 650 to $680^{\circ} \mathrm{C}$ have been calculated for a pressure of $20 \mathrm{~kb}$ (Table 7).

Previous authors have estimated the $P-T$ conditions of the eclogite-facies metamorphism in the Champtoceaux nappe using assemblages from eclogites (Godard et al., 1981; Paquette et al., 1985) and quartzites (Ballèvre et al., 1987). Temperatures of the order of $700^{\circ} \mathrm{C}$ have been calculated using Fe-Mg partitioning between garnet and omphacite (Ellis \& Green, 1979). Pressures were found to be in the range 16-20 kb (Godard et al., 1981) or 18-20 kb (Ballèvre et al., 1987). Garnet-phengite thermometry in the studied micaschists gives slightly lower temperatures than these previous estimates. This discrepancy probably results from uncertainties in the $\mathrm{Fe}^{3+}$ content.

In conclusion, estimated $P-T$ conditions for the eclogitic metamorphism are $650-700^{\circ} \mathrm{C}$ at pressures ranging from 10 to $20 \mathrm{~kb}$.

\section{$\mathrm{P}-\mathrm{T}$ evolution of the Champtoceaux micaschists}

As shown previously, the Champtoceaux micaschists preserve part of their prograde $P-T$ path. A first step towards quantitative estimates is shown in Fig. 12, where phase relations 


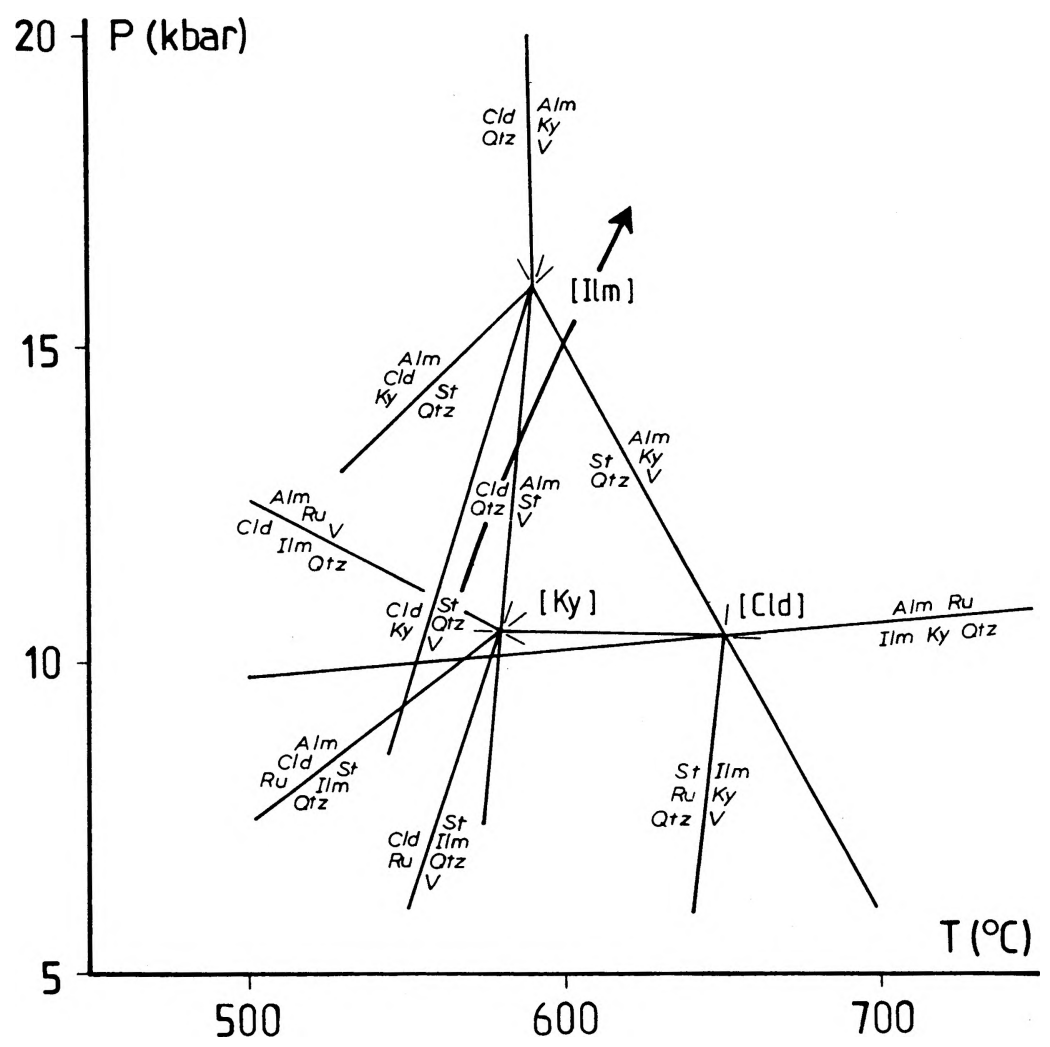

FIG. 12. Phase relations in the $\mathrm{FeO}-\mathrm{Al}_{2} \mathrm{O}_{3}-\mathrm{TiO}_{2}-\mathrm{SiO}_{2}-\mathrm{H}_{2} \mathrm{O}$ system. Mineral abbreviations are listed in Table 1. Only quartz-bearing reactions are represented. The $\mathrm{St}+\mathrm{Ilm}+\mathrm{Qtz}(\mathrm{Ky}, \mathrm{Cld}) \mathrm{Alm}+\mathrm{Rt}+\mathrm{H}_{2} \mathrm{O}$ is not labelled. The arrow shows the prograde $\boldsymbol{P}-\boldsymbol{T}$ path as recorded by mineral assemblages during garnet growth.

between almandine, chloritoid, staurolite, kyanite, rutile, and ilmenite with excess quartz and vapour are depicted in the $\mathrm{FeO}-\mathrm{Al}_{2} \mathrm{O}_{3}-\mathrm{TiO}_{2}-\mathrm{SiO}_{2}-\mathrm{H}_{2} \mathrm{O}$ system. Experimental data allow the location of some reaction curves:

(1) According to Rao \& Johannes (1979), the [Ilm] invariant point is located at around $590^{\circ} \mathrm{C}$ and $16 \mathrm{~kb}$. Three of the five curves emanating from this invariant point have been experimentally studied (Richardson, 1968; Ganguly, 1972; Rao \& Johannes, 1979):

$$
\begin{gathered}
4 \mathrm{Fe}_{-} \mathrm{Cld}+5 \mathrm{Ky}=2 \mathrm{Fe}_{-} \mathrm{St}+1 \cdot 5 \mathrm{Qtz}+2 \mathrm{H}_{2} \mathrm{O} \\
23 \mathrm{Fe}_{-} \mathrm{Cld}+7 \mathrm{Qtz}=4 \mathrm{Fe}_{-} \mathrm{St}+5 \mathrm{Alm}+19 \mathrm{H}_{2} \mathrm{O} \\
6 \mathrm{Fe} \_\mathrm{St}+12 \cdot 5 \mathrm{Qtz}=4 \mathrm{Alm}+23 \mathrm{Ky}+6 \mathrm{H}_{2} \mathrm{O}
\end{gathered}
$$

(2) Bohlen et al. (1983) studied the $P-T$ location of the reaction:

$$
\mathrm{Alm}+3 \mathrm{Rt}=3 \mathrm{Ilm}+\mathrm{Ky}+2 \mathrm{Qtz}
$$

and showed that the [Cld] invariant point is situated at around $11 \mathrm{~kb}, 650^{\circ} \mathrm{C}$.

Without an internally consistent set of thermodynamic data on end-member phases, we have been unable to calculate the exact position of the univariant reaction curves. Moreover, observed phases in the Champtoceaux micaschists are not purely ferrous. Consequently, 
univariant reactions of the model system are divariant equilibria in the $\mathrm{FeO}-\mathrm{MgO}-\mathrm{Al}_{2} \mathrm{O}_{3}-\mathrm{TiO}_{2}-\mathrm{SiO}_{2}-\mathrm{H}_{2} \mathrm{O}$ system. Despite these uncertainties, the model system provides useful constraints on the prograde $P-T$ path.

Indeed, the lack of ilmenite and kyanite and the presence of chloritoid and staurolite within garnet cores show that the prograde $P-T$ path took place along curve (12), at pressures between the $[\mathrm{Ky}]$ and $[\mathrm{llm}]$ invariant points. Equilibrium (8) was exceeded when staurolite broke down and kyanite grew (garnet rims). This shows that garnet growth occurred during a strong pressure increase while temperature rose slightly, and suggests that the matrix assemblages equilibrated at pressures higher than $16 \mathrm{~kb}$.

\section{Geological significance of the $\mathrm{P}-\mathrm{T}$ history}

Figure 13 shows a part of the $P-T$ history of the lower unit of the Champtoceaux nappe. A detailed discussion of the entire $P-T$ history is clearly beyond the scope of this paper, because the last stages of the uplift path are not well constrained and other units within the Champtoceaux nappe (Fig. 1) have suffered different $P-T$ histories during the Variscan orogeny. It thus seems premature to discuss only a particular $P-T$ history - that of the

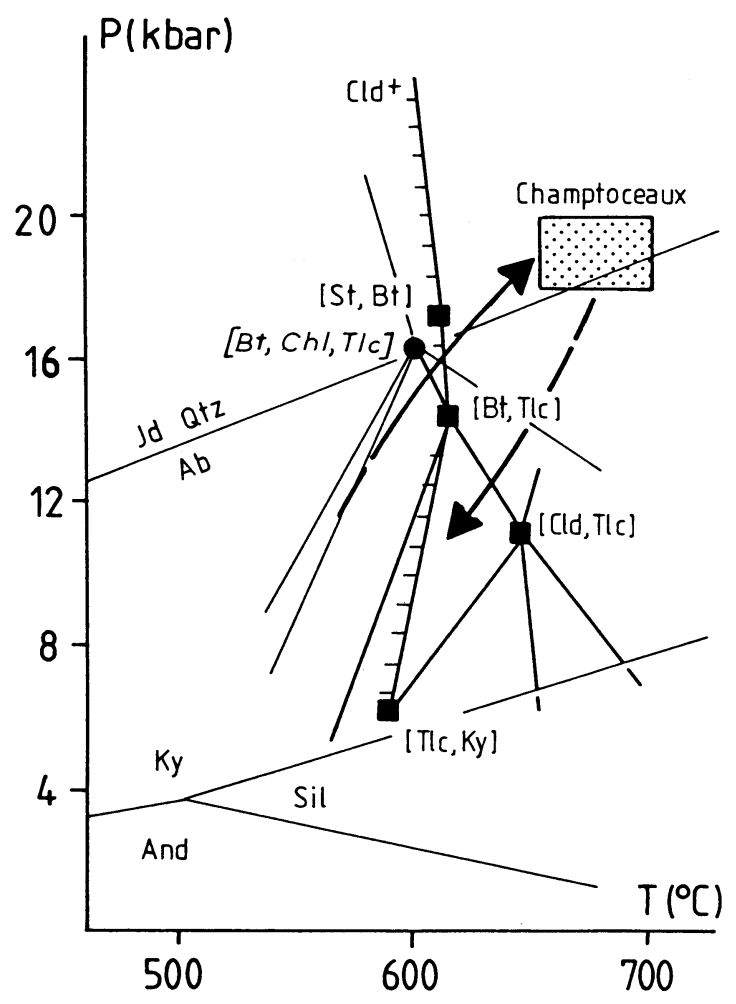

FIG. 13. Pressure-temperature history of the Champtoceaux metapelites. Alumino-silicate phase relations after Holdaway (1971). Ab $=\mathrm{Jd}+\mathrm{Qtz}$ after Holland (1980). The KFMASH multisystem of Fig. 10b is approximately located on the $P-T$ diagram after Rao \& Johannes (1979), Koons \& Thompson (1985) and Vuichard \& Ballèvre (1988). Upper limit of the chloritoid stability field is indicated $\left(\mathrm{Cld}^{+}\right)$. Shaded area represents peak metamorphic conditions for the Champtoceaux nappe. Arrows show $P-T$ path for the Champtoceaux. 
studied unit - without reference to lower and higher structural levels of the same nappe pile. Some comments on the $\boldsymbol{P}-\boldsymbol{T}$ history are nevertheless useful:

(1) In the unit studied, eclogite-facies metamorphism is recorded not only in the metabasites but also in pelitic and quartzitic sediments (Ballèvre et al., 1987). Incomplete coronitic transformations observed within low-strained granitic areas (Lasnier et al., 1973) can also be attributed to the eclogite-facies metamorphism. Thus the studied unit should no longer be considered as a 'tectonic melange' (e.g., Marchand, 1981; Godard, 1983).

(2) The lack of relict parageneses from a previous metamorphic event suggests that the studied unit was most probably submitted to a single metamorphic cycle. For example, such relicts are frequently observed in the Western Alps, where a Paleozoic continental basement was submitted to an eclogite-facies metamorphism during the Cretaceous Eoalpine events (e.g., Compagnoni, 1977; Dal Piaz \& Lombardo, 1986). In the present example, however, the breakdown of the graphitic material observed in the metapelites during the prograde history (Figs. 2 and 3) argues for a single-phase metamorphic history and therefore for pelitic sediments of Lower Paleozoic age.

(3) Consequently, the lower unit of the Champtoceaux nappe is a fratgment of the upper levels of the continental crust, which was buried to depths of $c a .60 \mathrm{~km}$ at an early stage of the Variscan events. This shows that crustal thickening was a major process during the Variscan orogeny, which is consistent with present models of the tectonic evolution of the whole belt (e.g., Matte, 1986).

(4) The $P-T$ path of the studied unit is mainly characterized by the fact that the maximum temperature is achieved at a depth equal to the maximum amount of burial (Fig. 13). This is the only known example of such a $P-T$ path in the Variscan belt. This kind of $P-T$ path is not consistent with most simple one-dimensional thermal models (England \& Richardson, 1977; England \& Thompson, 1984), except those obtained with high uplift rates, e.g., in the case where extension followed thickening (Thompson \& Ridley, 1987), or those calculated with normal uplift rates but where thermal re-equilibration is cancelled by screen effects (Rubie, 1984; Davy \& Gillet, 1986). Clearly, more data on the $P-T$ evolution of the whole Champtoceaux nappe are needed to constrain possible thermal-tectonic models.

\section{CONCLUSIONS}

The main conclusions of this study are as follows:

(1) A reversal of $\mathrm{Fe}-\mathrm{Mg}$ partitioning occurs in high-pressure metapelites, where staurolite is more Fe-rich than coexisting garnet (Fig. 7). Only three natural occurrences are known at present (in Kazakhstan in the USSR, the Dora-Maira nappe in the Western Alps, and the Champtoceaux nappe in Brittany) but careful examination of garnet-kyanite-phengite-rutile micaschists world-wide may reveal further examples.

(2) Taking into account the $\mathrm{Fe}-\mathrm{Mg}$ reversal between garnet and staurolite, we analyse the phase relations between garnet, chloritoid, staurolite, and kyanite in eclogitic metapelites. The resulting topology of univariant reactions in the KFMASH multisystem is depicted in Fig. 10b, and compositional changes are predicted by Fig. $11 \mathrm{~b}$.

(3) The Champtoceaux micaschists record a trend of increasing pressures and temperatures towards 'peak' metamorphic conditions which have been estimated at around $18-20 \mathrm{~kb}, 650-700^{\circ} \mathrm{C}$. During this prograde evolution, mineralogical and compositional changes are controlled by the KFMASH univariant reaction:

$$
\mathrm{St}+\mathrm{Cld}=\mathrm{Grt}+\mathrm{Ky} \text {. }
$$


(4) The lower unit of the Champtoceaux nappe (Brittany, France) records burial of continental basement to depths of $c a .60 \mathrm{~km}$ during the early stages of the Variscan orogeny.

\section{ACKNOWLEDGEMENTS}

Many thanks are due to Philippe Bertrand for his helpful suggestions during the course of this work. Critical reviews by S. L. Harley and N. B. W. Harris were greatly appreciated. M. S. N. Carpenter helped us to improve the English text.

\section{REFERENCES}

Albee, A. L., 1972. Metamorphism of pelitic schists: reaction relations of chloritoid and staurolite. Geol. Soc. Am. Bull. 83, 3249-68.

Ballèvre, M., Kienast, J. R., \& Paquette, J. L., 1987. Le métamorphisme éclogitique dans la nappe hercynienne de Champtoceaux (Massif Armoricain). C. R. Acad. Sci. Paris 305-II, 127-31.

Bohlen, S. R., Wall, V. J., \& Boettcher, A. L., 1983. Experimental investigations and geological applications of equilibria in the system $\mathrm{FeO}-\mathrm{TiO}_{2}-\mathrm{Al}_{2} \mathrm{O}_{3}-\mathrm{SiO}_{2}-\mathrm{H}_{2} \mathrm{O}$. Am. Miner. 68, 1049-58.

Brière, Y., 1920. Les éclogites françaises. Leur composition minéralogique et chimique; leur origine. Bull. Soc. Fr. Miner. 43, 71-222.

Brown, E. H., \& Forbes, R. B., 1986. Phase petrology of eclogitic rocks in the Fairbanks district, Alaska. Geol. Soc. Am. Mem. 164, 155-67.

Chopin, C., 1985. Les relations de phases dans les métapélites de haute pression. Thèse de Doctorat d'État, Université de Paris VI, $80 \mathrm{pp}$.

- Schreyer, W., 1983. Magnesiocarpholite and magnesiochloritoid: two index minerals of pelitic blueschists in the model system $\mathrm{MgO}-\mathrm{Al}_{2} \mathrm{O}_{3}-\mathrm{SiO}_{2}-\mathrm{H}_{2}$ O. Am. J. Sci. 283-A, 72-92.

Compagnoni, R., 1977. The Sesia-Lanzo zone: high-pressure-low-temperature metamorphism in the Austroalpine continental margin. Rend. Soc. Ital. Miner. Petrol. 33, 335-74.

Dal Piaz, G. V., \& Lombardo B., 1986. Early-Alpine eclogite metamorphism in the Penninic Monte Rosa-Gran Paradiso basement nappes of the northwestern Alps. Geol. Soc. Am. Mem. 164, 249-65.

Davy, P., \& Gillet, P., 1986. The stacking of thrust slices in collision zones and its thermal consequences. Tectonics 5, 913-29.

Delor, C., Burg, J. P., Guiraud M., \& Leyreloup A., 1987. Les métapélites à phengite-chloritö̈degrenat-staurotide-disthène de la klippe de Najac-Carmaux: nouveaux marqueurs d'un métamorphisme de haute pression varisque en Rouergue occidental. C. R. Acad. Sci. Paris 305-II, 589-95.

Droop, G. T. R., \& Bucher-Nurminen, K., 1984. Reaction textures and the metamorphic evolution of sapphirinebearing granulites from the Gruf Complex, Italian. Central Alps. J. Petrology 25, 766-803.

Ellis, D. J., \& Green D. H., 1979. An experimental study on the effect of Ca upon garnet-clinopyroxene Fe-Mg exchange equilibria. Contr. Miner. Petrol. 71, 13-22.

Enami, M., \& Zang, Q., 1988. Magnesian staurolite in garnet-corundum rocks and eclogite from the Donghai district, Jiangsu province, east China. Am. Miner. 73, 48-56.

England, P. C., \& Richardson, S. W., 1977. The influence of erosion upon the mineral facies of rocks from different metamorphic environments. J. Geol. Soc. London 134, 201-13.

- Thompson, A. B., 1984. Pressure-temperature-time paths of regional metamorphism. I: Heat transfer during the evolution of thickened continental crust. J. Petrology 25, 894-928.

Ferry, J. M., \& Spear, F. S., 1978. Experimental calibration of the partitioning of Fe and $\mathrm{Mg}$ between biotite and garnet. Contr. Miner. Petrol. 66, 113-17.

Ganguly, J., 1972. Staurolite stability and related parageneses: theory, experiments, and applications. J. Petrology $13,335-65$.

Gibson, R. G., \& Speer, J. A., 1986. Contact aureoles as constraints on regional $P-T$ trajectories: an example from the Northern Alabama Piedmont, USA. J. Metamorphic Geol. 4, 285-308.

Godard, G., 1983. Dispersion tectonique des éclogites de Vendée lors d'une collision continent-continent. Bull. Miner. 106, 719-22.

Kienast, J. R., \& Lasnier, B., 1981. Retromorphic development of glaucophane in some eclogites, east of Nantes (Massif Armoricain, France). Contr. Miner. Petrol. 78, 126-35.

Goffé, B., \& Chopin, C., 1986. High-pressure metamorphism in the Western Alps: zoneography of metapelites, chronology and consequences. Schweiz. Miner. Petrogr. Mitt. 66, 41-52.

Grambling, J. A., 1983. Reversals in Fe-Mg partitioning between chloritoid and staurolite. Am. Miner. 68, $373-88$.

Green, T. H., \& Hellman, P. L., 1982. Fe-Mg partitioning between coexisting garnet and phengite at high pressure, and comments on a garnet-phengite geothermometer. Lithos 15, 253-66.

Greenwood, H. J., 1975. Thermodynamically valid projections of extensive phase relationships. Am. Miner. 60, 1-8.

Grew, E. S., \& Sandiford, M., 1984. A staurolite-talc assemblage in tourmaline-phlogopite-chlorite schist from northern Victoria Land, Antartica, and its petrologic significance. Contr. Miner. Petrol. 87, 337-50. 
Griffen, D. T., Gosney, T. C., \& Revell Phillips, W. M., 1982. The chemical formula of natural staurolite. Am. Miner. 67, 292-7.

Guidotti, C. V., 1974. Transition from staurolite to sillimanite zone, Rangely Quadrangle, Maine. Geol. Soc. Am. Bull. 85, 475-90.

Harte, B., \& Hudson, N. F. C., 1979. Pelite facies series and the temperature and pressure of the Dalradian metamorphism in E. Scotland. In: The Caledonides of the British Isles-Reviewed. Geol. Soc. Lond. Spec. Publ., 8, 323-37.

Hensen, B. J., 1986. Theoretical phase relations involving cordierite and garnet revisited: the influence of oxygen fugacity on the stability of sapphirine and spinel in the system $\mathrm{Mg}-\mathrm{Fe}-\mathrm{Al}-\mathrm{Si}-\mathrm{O}$. Contr. Miner. Petrol. 92, $362-7$.

Holdaway, M. J., 1971. Stability of andalusite and the aluminium silicate phase diagram. Am. J. Sci. 271, 97-131.

1978. Significance of chloritoid-bearing and staurolite-bearing rocks in the Picuris Range, New Mexico..Geol. Soc. Am. Bull. 89, 1404-14.

_ Dutrow, B. L., Borthwhick, J., Shore, P., \& Harmon, R. S., 1986a. H content of staurolite as determined by H extraction line and ion microprobe. Am. Miner. 71, 1135-41.

Shore, P., 1986b. A model for the crystal chemistry of staurolite. Ibid. 71, 1142-59.

Hinton, R. W., 1988. Devonian and Carboniferous metamorphism in west-central Maine: the muscovite-almandine geobarometer and the staurolite problem revisited. Ibid. 73, $20-47$.

Holland, T. J. B., 1979. Experimental determination of the reaction paragonite $=$ jadeite + kyanite $+\mathrm{H}_{2} \mathrm{O}$, and internally consistent thermodynamic data for part of the system $\mathrm{Na}_{2} \mathrm{O}-\mathrm{Al}_{2} \mathrm{O}_{3}-\mathrm{SiO}_{2}-\mathrm{H}_{2} \mathrm{O}$, with applications to eclogites and blueschists. Contr. Miner. Petrol. 68, 293-301.

- 1980. The reaction albite $=$ jadeite + quartz determined experimentally in the range $600-1200^{\circ} \mathrm{C}$. Am. Miner. 65, 129-34.

Klaper, E. M., \& Bucher-Nurminen, K., 1987. Alpine metamorphism of pelitic schists in the Nufenen Pass area, Lepontine Alps. J. Metamorphic Geol. 5, 175-94.

Koons, P. O., \& Thompson, A. B., 1985. Non-mafic rocks in the greenschist, blueschist and eclogite facies. Chem. Geol. 50, 3-30.

Lacroix, A., 1891. Etude pétrologique des éclogites de la Loire infërieure. Bull. Soc. Sci. Nat. Ouest Fr. 1, 81-114.

Lagarde, J. L., 1978. La déformation des roches dans les domaines à schistosité subhorizontale (Champtoceaux-Canigou Roc de France). Thèse de Doctorat de 3ème Cycle, Université de Rennes, $164 \mathrm{pp}$.

Lang, H. M., \& Rice, J. M., 1985. Geothermometry, geobarometry and $T-X(\mathrm{Fe}-\mathrm{Mg})$ relations in metapelites, Snow Peak, Northern Idaho. J. Petrology 26, 889-924.

Lasnier, B., Leyreloup, A., \& Marchand, J., 1973. Découverte d'un granite 'charnockitique' au sein des 'gneiss oeillés'. Perspectives nouvelles sur l'origine de certaines leptynites du Massif Armoricain méridional (France). Contr. Miner. Petrol. 41, 131-44.

Leupolt, L., \& Franz, G., 1986. Zink-Staurolith aus Metasedimenten der Eclogitzone, Tauern/Osterreich. Fortschr. Miner. 64, 96 (abstract).

Loomis, T. P., 1983. Compositional zoning of crystals: a record of growth and reaction history. In Saxena, S. K. (ed.) Kinetics and Equilibrium in Mineral Reactions. Advances in Physical Geochemistry, Vol. 3. Heidelberg: Springer-Verlag, $1-60$.

Marchand, J., 1981. Ecaillage d'un 'mélange tectonique' profond: le complexe cristallophyllien de Champtoceaux (Bretagne méridionale). C. R. Acad. Sci. Paris 293-II, 223-7.

1983. Légende de la carte géologique de la France, feuille de Nort-sur-Erdre. Orléans: Bur. Rech. Geol. Min.

Massone, H. J., \& Schreyer, W., 1987. Phengite geobarometry based on the limiting assemblage with K-feldspar, phlogopite, and quartz. Contr. Miner. Petrol. 96, 212-24.

Matte, P, 1986. Tectonics and plate tectonics model for the Variscan belt of Europe. Tectonophysics 126, 329-74.

Paquette, J. L., 1987. Comportement des systèmes isotopiques U-Pb et $\mathrm{Sm}-\mathrm{Nd}$ dans le métamorphisme éclogitique. Chaîne hercynienne et chaîne alpine. Docum. Centre Armor. Et. Struc. Socles, Rennes, 14, 190 pp.

— Marchand, J., \& Peucat, J. J., 1984. Absence de tectonique cadomienne dans le complexe de Champtoceaux (Bretagne méridionale)? Comparaison des systèmes $\mathrm{Rb}-\mathrm{Sr}$ et U-Pb d'un métagranite. Bull. Soc. Geol. Fr. XXVI-5, 907-12.

_ Peucat, J. J., Bernard-Griffiths, J., \& Marchand, J., 1985. Evidence for old Precambrian relics shown by U-Pb zircon dating of eclogites and associated rocks in the Hercynian belt of South Brittany, France. Chem. Geol. 52, 203-16.

Pigage, L. C., \& Greenwood, H. J., 1982. Internally consistent estimates of pressure and temperature: the staurolite problem. Am. J. Sci. 282, 943-69.

Rao, B. B., \& Johannes, W., 1979. Further data on the stability of staurolite + quartz and related assemblages. $N$. Jb. Miner. Mh. H10, 437-47.

Rice, J. M., 1985. Experimental partitioning of Fe and Mg between coexisting staurolite and garnet. EOS 66, 1127 (abstract).

Richardson, S. W., 1968. Staurolite stability in a part of the system Fe-Al-Si-O-H. J. Petrology 9, 467-88.

Rubie, D. C., 1984. A thermal-tectonic model for high-pressure metamorphism and deformation in the Sesia zone, Western Alps. J. Geol. 92, 21-36.

Schreyer, W., 1968. A reconnaissance study of the system $\mathrm{MgO}-\mathrm{Al}_{2} \mathrm{O}_{3}-\mathrm{SiO}_{2}-\mathrm{H}_{2} \mathrm{O}$ at pressures between 10 and 25 kb. Carnegie Inst. Wash. Yb. 66, 380-92. 
Horrocks, P. C., \& Abraham, K., 1984. High-magnesium staurolite in a sapphirine-garnet rock from the Limpopo Belt, Southern Africa. Contr. Miner. Petrol. 86, 200-7.

Smith, D. C., 1984. Remarques cristallochimiques et petrogénétiques sur des minéraux inhabituels dans les éclogites de Liset et Rekvika, Norvège. Dixième Réunion Annuelle des Sciences de la Terre, Bordeaux, 1984. Paris: Société Géologique de France, 511 (abstract).

Spear, F. S., \& Franz, G., 1986. $P-T$ evolution of metasediments from the eclogite zone, south-central Tauern Window, Austria. Lithos 19, 219-34.

Spry, A., 1969. Metamorphic Textures. Oxford: Pergamon Press, 350 pp.

Thompson, A. B., \& Ridley, J. R., 1987. Pressure-temperature-time $(P-T-t)$ histories of orogenic belts. Phil. Trans. R. Soc. Lond., Ser. A, 321, 27-45.

Thompson, J. B., Jr., 1957. The graphical analysis of mineral assemblages in pelitic schists. Am. Miner. 42, 842-58.

Udovkina, N. G., Muravistkaya, G. N., \& Laputina, I. P., 1980. Talc-garnet-kyanite rocks of the Kokchetav block, Northern Kazakhstan. Dokl. Earth Sci. Sect. 237, 202-5.

Velde, B., 1966. Etude minéralogique d'une éclogite de Fay-de-Bretagne (Loire Atlantique). Bull. Soc. Fr. Miner. Cristall. 89, 385-93.

- 1970. Les éclogites de la région nantaise (de Campbon au Cellier, Loire Atlantique). Ibid. 93, 370-85.

Vidal, Ph., Peucat, J. J., \& Lasnier, B., 1980. Dating of granulites involved in the Hercynian fold-belt of Europe: an example taken from the granulite-facies orthogneiss at La Picherais, Southern Armorican Massif, France. Contr. Miner. Petrol. 72, 283-9.

Vuichard, J. P., \& Ballèvre, M., 1988. Garnet-chloritoid equilibria in eclogitic pelitic rocks from the Sesia zone (Western Alps): their bearing on phase relations in high pressure metapelites. J. Metamorphic Geol. 6, 135-57.

Ward, C. M., 1984. Magnesium staurolite and green chromian staurolite from Fiordland, New Zealand. Am. Miner. 69, 531-40.

Yardley, B. W. D., Leake, B. E., \& Farrow, C. M., 1980. The metamorphism of Fe-rich pelites from Connemara, Ireland. J. Petrology 21, 365-99. 